quatrième série-tome $46 \quad$ fascicule $4 \quad$ juillet-aồt 2013

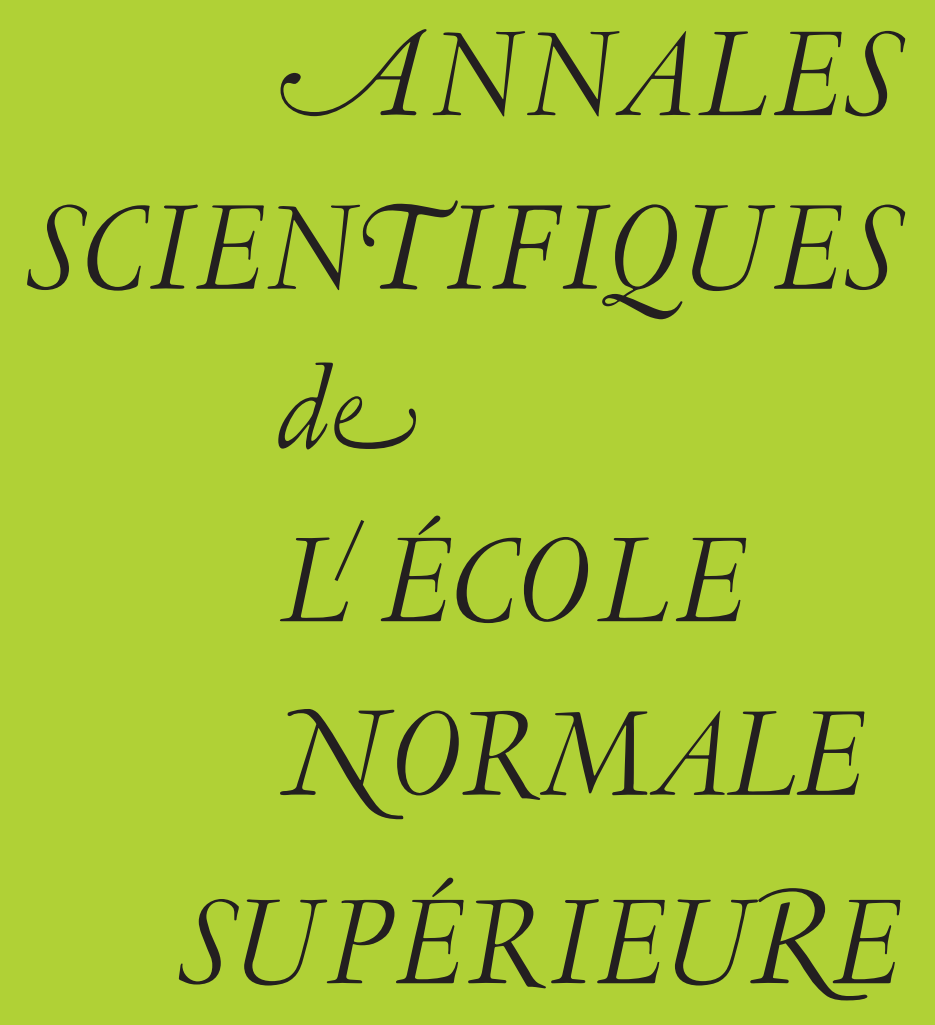

Mikhail BOROVOI \& Cyril DEMARCHE \& David HARARI

Complexes de groupes de type multiplicatif

et groupe de Brauer non ramifié

des espaces bomogènes

SOCIÉTÉ MATHÉMATIQUE DE FRANCE 
Ann. Scient. Éc. Norm. Sup.

$4^{\text {e }}$ série, t. 46, 2013, p. 651 à 692

\section{COMPLEXES DE GROUPES \\ DE TYPE MULTIPLICATIF \\ ET GROUPE DE BRAUER NON RAMIFIÉ \\ DES ESPACES HOMOGÈNES}

\section{PAR Mikhail BOROVOI, CYRIL DEMARCHE ET David HARARI}

RÉSUMÉ. - On calcule par des méthodes arithmétiques le groupe de Brauer non ramifié des espaces homogènes de groupes algébriques linéaires sur différents corps. Les formules obtenues font intervenir l'hypercohomologie de complexes de groupes de type multiplicatif.

Abstract. - We compute by arithmetic methods the unramified Brauer group of homogeneous spaces of linear algebraic groups over various fields. We get formulae in terms of hypercohomology of complexes of groups of multiplicative type.

\section{Introduction}

Soit $G$ un groupe algébrique linéaire connexe lisse défini sur un corps $k$. Soit $X$ un espace homogène de $G$, tel que le stabilisateur géométrique $\bar{H}$ soit de type (ssumult), c'est-à-dire une extension d'un groupe de type multiplicatif lisse par un groupe connexe, lisse et sans caractères (par exemple, un groupe linéaire lisse et connexe sur un corps parfait est de type (ssumult), ainsi qu'un groupe linéaire commutatif sur un corps de caractéristique zéro). Le but de cet article est d'établir des formules (obtenues précédemment dans des cas particuliers par Colliot-Thélène et Kunyavskiı̌ [15], [16]) pour le groupe de Brauer algébrique $\operatorname{Br}_{1} X^{c}$ (et parfois pour tout le groupe de Brauer $\mathrm{Br} X^{c}$ ) d'une compactification lisse $X^{c}$ de $X$. Le groupe $\mathrm{Br} X^{c}$ coïncide avec le groupe de Brauer non ramifié de $X$ si $k$ est de caractéristique zéro (et le même énoncé vaut en caractéristique $p$ exception faite de la torsion $p$-primaire).

Dans le cas où $k$ est un corps global, on rappelle que ce groupe $\operatorname{Br} X^{c}$ intervient de façon cruciale dans l'étude de l'arithmétique de l'espace homogène $X$ et de sa compactification $X^{c}$, via l'obstruction de Brauer-Manin au principe de Hasse et à l'approximation faible (voir par exemple [48], théorème 5.2.1 (a)). Par ailleurs, ce groupe est également utilisé pour étudier la rationalité de l'espace homogène $X$ sur un corps $k$ quelconque (voir par exemple [17]). Ces deux exemples illustrent l'intérêt et la motivation que l'on peut avoir à disposer de formules décrivant le groupe $\mathrm{Br} X^{c}$ sur un corps $k$ quelconque. 
Comme dans les travaux antérieurs, les formules obtenues ici pour le groupe $\mathrm{Br} X^{c}$ font intervenir des groupes « $\amalg_{\omega}$ ", mais ils sont associés à des groupes d'hypercohomologie galoisienne de certains complexes (définis et utilisés dans [9] et [10]) au lieu simplement de groupes de cohomologie galoisienne.

Les résultats principaux de cet article sont les suivants :

1. Soient $k$ un corps algébriquement clos de caractéristique zéro et $X=G / H$ un espace homogène d'un groupe algébrique linéaire connexe $G$, avec $H$ connexe. Alors (théorème 5.1) le groupe de Brauer $\operatorname{Br} X^{c}$ d'une compactification lisse $X^{c}$ de $X$ est nul. Ceci étend le résultat classique de Bogomolov (qui concerne le cas $k=\mathbf{C}$ et $G$ semi-simple simplement connexe), et répond par l'affirmative à une question posée par Colliot-Thélène et Kunyavskiī (et également auparavant par Colliot-Thélène et Sansuc). Si $k$ est séparablement clos de caractéristique $p$, le même résultat vaut pour la partie première à $p \operatorname{de~} \operatorname{Br} X^{c}$ si l'on suppose de plus $G$ et $H$ lisses et réductifs.

2. Pour un espace homogène $X$ d'un groupe linéaire connexe $G$ sur un corps $k$ quelconque de caractéristique zéro, on obtient une formule « de type $\amalg_{\omega}$ » pour le groupe de Brauer algébrique $\mathrm{Br}_{1} X^{c}$, qui est valable dès que le stabilisateur géométrique $\bar{H}$ est de type (ssumult) et $G$ est de groupe de Picard géométrique nul (théorème 8.1). Ce résultat généralise le théorème principal de [16] dans deux directions : on ne demande plus que $G$ soit quasi-trivial, et l'hypothèse sur $\bar{H}$ est plus faible que l'hypothèse habituelle de connexité (sous laquelle notre théorème 5.1 permet également d'obtenir la formule pour tout le groupe de Brauer $\mathrm{Br} X^{c}$ et pas seulement pour $\mathrm{Br}_{1} X^{c}$ ). On a de plus des contre-exemples qui montrent que l'hypothèse que $\bar{H}$ est de type (ssumult) ne peut pas être supprimée; en particulier l'hypothèse que le groupe des composantes connexes de $\bar{H}$ est commutatif n'est pas suffisante (proposition 8.4).

3. On montre également une formule analogue sur un corps global de caractéristique $p$ (théorème 7.4), et on prouve la trivialité du groupe considéré sur un corps fini (théorème 7.5) moyennant quelques hypothèses de lissité additionnelles. Noter aussi que l'utilisation d'un théorème récent de Gabber (qui précise le théorème d' A. J. de Jong sur les altérations) permet de démontrer la plupart de ces énoncés sur $\operatorname{Br}_{n r} X$ en caractéristique $p$ (en exceptant la torsion $p$-primaire) sans supposer l'existence d'une compactification lisse pour $X$.

Une autre nouveauté importante de cet article est que la méthode de démonstration de plusieurs de nos théorèmes passe (comme pour le résultat principal de [15]) par le cas des corps finis, mais pour traiter ceux-ci on est amené à passer sur des corps locaux de caractéristique $p$ et à utiliser des théorèmes de dualité arithmétique pour obtenir le résultat dans ce contexte. C'est pourquoi on a été amené à étendre certains résultats classiques de dualité sur les corps $p$-adiques aux corps locaux de caractéristique quelconque, et aussi à certains complexes de groupes de type multiplicatif au lieu de se limiter à des modules galoisiens (proposition 3.2, proposition 7.2). Enfin on donne aussi au passage une démonstration détaillée d'une formule de compatibilité entre accouplements (théorème 6.2) qui est utile en elle-même, notamment si on est intéressé par l'accouplement de Brauer-Manin sur les espaces homogènes au-dessus d'un corps global.

4 e SÉRIE - TOME $46-2013-$ No $^{\circ}$ 


\section{Notations et conventions}

Dans ce texte, tous les schémas en groupes sont supposés localement de type fini. Si $k$ est un corps, on dit simplement « $k$-groupe» pour désigner un $k$-schéma en groupes linéaire, i.e. affine et de type fini. Un $k$-groupe réductif (resp. semi-simple) est un $k$-schéma en groupes affine, lisse, connexe, et réductif (resp. semi-simple). On note souvent $G^{0}$ la composante connexe du neutre d'un $k$-groupe $G$. Si $G$ est un $k$-groupe connexe lisse sur un corps parfait, on note $G^{\mathrm{u}}$ son radical unipotent, $G^{\text {red }}:=G / G^{\mathrm{u}}$ le quotient réductif, $G^{\text {ss }}$ le sous-groupe dérivé de $G^{\text {red }}$ et $G^{\text {sc }}$ le revêtement semi-simple simplement connexe de $G^{\text {ss }}$. On dit alors que $G$ est quasi-trivial si $G^{\text {tor }}:=G^{\text {red }} / G^{\text {ss }}$ est quasi-trivial (i.e. son groupe des caractères est un module galoisien de permutation) et $G^{\text {ss }}$ est simplement connexe.

Pour tout corps $k$, on note $\bar{k}$ une clôture séparable de $k$ et $\Gamma_{k}=\operatorname{Gal}(\bar{k} / k)$. Si $X$ est un $k$-schéma et $L$ une extension de $k$, on pose $X_{L}:=X \times_{k} L$ et $\bar{X}=X \times_{k} \bar{k}$. Les groupes de cohomologie étale $H_{\mathrm{et}}^{n}(X, \ldots)$ sont notés simplement $H^{n}(X, \ldots)$, tandis qu'on note $H_{\mathrm{fppf}}^{n}(X, \ldots)$ s'il s'agit de groupes fppf (les deux coïncident pour des faisceaux représentés par des schémas en groupes lisses et quasi-projectifs). Pour simplifier on note aussi souvent $H^{n}\left(X, C^{\bullet}\right)$ les groupes d'hypercohomologie $\mathbf{H}^{n}\left(X, C^{\bullet}\right)$ quand $C^{\bullet}$ est un complexe borné de faisceaux étales sur $X$ (et de même avec les groupes fppf). On adopte des notations analogues pour les ensembles de cohomologie (non abélienne) $H^{1}(X, \ldots)$. En particulier si $G$ est un $k$-schéma en groupes lisse, l'ensemble $H^{1}(k, G)$ (resp. les groupes $H^{i}(k, G)$ si $G$ est commutatif) s'identifie à l'ensemble de cohomologie galoisienne $H^{1}\left(\Gamma_{k}, G(\bar{k})\right)$ (resp. aux groupes de cohomologie galoisienne $\left.H^{i}\left(\Gamma_{k}, G(\bar{k})\right)\right)$.

Par convention, quand on écrit un complexe à deux termes sous la forme $[A \rightarrow B]$, cela signifie que $A$ est en degré -1 et $B$ en degré 0 . Si $Z$ est un schéma, on note $\mathscr{D}(Z)$ (resp. $\mathscr{D}_{\mathrm{fppf}}(Z)$ ) la catégorie dérivée bornée des faisceaux étales (resp. fppf) sur $Z$. Si $k$ est un corps et $M$ est un $\Gamma_{k}$-module galoisien (ou encore un complexe borné de modules galoisiens), on note (pour tout $i \geq 1$ ) $\amalg_{\omega, \text { alg }}^{i}(k, M)$ (ou $\amalg_{\omega, \text { alg }}^{i}(M)$ s'il n'y a pas d'ambiguïté sur le corps $k$ ) le sous-groupe de $H^{i}\left(\Gamma_{k}, M\right)$ constitué des éléments dont la restriction à $H^{i}(C, M)$ est nulle pour tout sous-groupe procyclique $C$ de $\Gamma_{k}$.

Un corps local est un corps complet pour une valuation discrète à corps résiduel fini : c'est une extension finie de $\mathbf{Q}_{p}$ (s'il est de caractéristique 0) ou d'un corps de séries de Laurent $\mathbf{F}_{q}((t))$ sur un corps fini (s'il est de caractéristique $>0$ ). On utilisera fréquemment le fait (dû à Kneser [37] en caractéristique zéro et à Bruhat-Tits [11] en caractéristique $>0$ ) que pour un groupe semi-simple simplement connexe $G$ sur un corps local $K$, on a $H^{1}(K, G)=0$. Par Hilbert 90, on en déduit alors le résultat analogue si $G$ est quasi-trivial sur un corps local.

Un corps global est un corps de nombres (extension finie de $\mathbf{Q}$ ) ou le corps des fonctions d'une courbe algébrique sur un corps fini. Pour toute place $v$ d'un tel corps $k$, on note $k_{v}$ le complété de $k$ en $v$. Si $k$ est un corps global et $M$ un $\Gamma_{k}$-module galoisien (ou un complexe borné de modules galoisiens), on note $\amalg_{\omega}^{i}(k, M)$ (ou simplement $\amalg_{\omega}^{i}(M)$ ) le sous-groupe de $H^{i}(k, M)$ constitué des éléments dont la restriction à $H^{i}\left(k_{v}, M\right)$ est nulle pour presque toute place $v$ de $k$. Si de plus $M$ est un $\Gamma_{k}$-module galoisien de type fini (resp. de type fini sans torsion), une conséquence facile du théorème de Čebotarev est que $\amalg_{\omega}^{1}(M)=\amalg_{\omega, \text { alg }}^{1}(M)$ (resp. $\amalg_{\omega}^{2}(M)=\amalg_{\omega, \text { alg }}^{2}(M)$ ), [44], section 2 . 
Si $A$ est un groupe abélien, et $n \in \mathbf{N}, A[n]$ désigne le sous-groupe de $n$-torsion de $A$. Si $l$ est un nombre premier, $A\{l\}$ désigne le sous-groupe de torsion $l$-primaire de $A$, et $A\left\{l^{\prime}\right\}$ le sous-groupe de torsion première à $l$.

\section{Accouplement entre complexes de groupes de type multiplicatif}

Dans cette section on étend l'accouplement $S \times \widehat{S} \rightarrow \mathbf{G}_{m}$ entre un groupe de type multiplicatif $S$ et son groupe des caractères $\widehat{S}$ à des complexes à deux termes, et on montre un résultat de dualité lié à cet accouplement sur un corps local.

Lemme 3.1. - Soit $Z$ un schéma. Soient $C=[S \rightarrow T]$ un complexe de groupes de type multiplicatif de type fini sur $Z$ et $\widehat{C}=[\widehat{T} \rightarrow \widehat{S}]$ le complexe dual. Alors on a un accouplement canonique

$$
[S \rightarrow T] \otimes^{\mathbf{L}}[\widehat{T} \rightarrow \widehat{S}] \rightarrow \mathbf{G}_{m}[1]
$$

dans la catégorie dérivée bornée $\mathscr{D}_{\mathrm{fppf}}(Z)$ des faisceaux fppf sur $Z$. De plus, cet accouplement induit pour tout $n \geq 0$ un isomorphisme canonique

$$
H_{\text {fppf }}^{n}(Z,[S \rightarrow T]) \rightarrow \mathbf{R}^{n} \operatorname{Hom}_{Z}\left([\widehat{T} \rightarrow \widehat{S}], \mathbf{G}_{m}[1]\right)
$$

où $\operatorname{Hom}_{Z}(\ldots)$ désigne les homomorphismes dans la catégorie dérivée bornée $\mathscr{D}(Z)$ des faisceaux étales sur $Z$. La même assertion est valable si l'on remplace $\operatorname{Hom}_{Z}(\ldots)$ par $\operatorname{Hom}_{\mathscr{D}_{\mathrm{fppf}}(Z)}(\ldots)$.

Démonstration. - On note que par dualité pour les groupes de type multiplicatif, le complexe $\widehat{C}$ est le complexe des morphismes $\operatorname{Ham}^{*}\left(C, \mathbf{G}_{m}[1]\right)$ correspondant au foncteur «Hom interne» $\operatorname{Ham}\left(., \mathbf{G}_{m}[1]\right)$ dans la catégorie des complexes de faisceaux fppf sur $Z$. Alors $\left.\mathbf{R} \operatorname{Htam}\left(., \mathbf{G}_{m}[1]\right)\right)$ est le foncteur dérivé total de $\operatorname{H}\left(a m\left(., \mathbf{G}_{m}[1]\right)\right.$, d'où un morphisme naturel dans $\mathscr{D}_{\text {fppf }}(Z)$ :

$$
\widehat{C} \rightarrow \mathbf{R} \operatorname{tam}\left(C, \mathbf{G}_{m}[1]\right) .
$$

Par adjonction entre le produit tensoriel dérivé $\otimes^{\mathbf{L}}$ et $\mathbf{R} \mathcal{H}$ (am , on a un isomorphisme naturel d'adjonction (cf. [50], Th. 10.8.7) :

$$
\operatorname{Hom}_{\mathscr{D}_{\mathrm{fppf}}(Z)}\left(\widehat{C} \otimes^{\mathbf{L}} C, \mathbf{G}_{m}[1]\right)=\operatorname{Hom}_{\mathscr{D}_{\mathrm{fppf}}(Z)}\left(\widehat{C}, \mathbf{R} \mathcal{H} \operatorname{Cam}\left(C, \mathbf{G}_{m}[1]\right)\right)
$$

qui donne un morphisme

$$
\hat{C} \otimes \mathbf{L} C \rightarrow \mathbf{G}_{m}[1]
$$

fournissant l'accouplement souhaité.

Pour la deuxième assertion, on note pour commencer que le groupe de droite ne change pas que l'on travaille dans $\mathscr{D}(Z)$ ou dans $\mathscr{D}_{\text {fppf }}(Z)$ (via [48], lemme 2.3.7). L'isomorphisme se déduit immédiatement par dévissage à partir des cas extrêmes $S=0$ ou $T=0$ (via le lemme des cinq), le résultat étant alors connu (loc. cit.).

$4^{\text {e }}$ SÉRIE - TOME $46-2013-$ N $^{\circ} 4$ 
Remarque. - S'il n'y a pas d'hypothèse de lissité sur $S$ et $T$, il est important de considérer $H_{\mathrm{fppf}}^{n}(X,[S \rightarrow T])$, et non pas le groupe étale correspondant.

Quand $Z=$ Spec $k$ est le spectre d'un corps, le lemme 3.1 donne en particulier un accouplement de cup-produit

$$
H^{1}(k,[\widehat{T} \rightarrow \widehat{S}]) \times H_{\mathrm{fppf}}^{0}(k,[S \rightarrow T]) \rightarrow H_{\mathrm{fppf}}^{2}\left(k, \mathbf{G}_{m}\right)=H^{2}\left(k, \mathbf{G}_{m}\right)=\operatorname{Br} k
$$

Si $K$ est un corps local, on a une topologie naturelle sur les groupes $H_{\text {fppf }}^{i}(K,[S \rightarrow T])$ pour $i \geq 0$ : ce sont des groupes séparés (au sens de Hausdorff), localement compacts, dénombrables à l'infini, et totalement discontinus. Si $K$ est un corps local de caractéristique zéro, on obtient des groupes discrets pour $i \geq 1$ (et on peut définir la topologie sur $H_{\mathrm{fppf}}^{0}(K,[S \rightarrow T])$ via le fait que ce groupe est extension d'un quotient de $H^{0}(K, T)$ par un sous-groupe du groupe fini $H^{1}(K, S)$, cf. [22], section 3). Si $K$ est de caractéristique $p>0$, le groupe $H_{\mathrm{fppf}}^{1}(K, S)$ est en général infini (il est seulement profini) si $S$ contient de la $p$-torsion; la topologie sur les $H_{\text {fppf }}^{i}(K,[S \rightarrow T])$ est alors définie par le même procédé que dans [41], section III.6. On a alors l'énoncé suivant, dans l'esprit du théorème 3.1 de [22] :

Proposition 3.2. - Soit $K$ un corps local (de caractéristique quelconque). Soit $[S \rightarrow T]$ un complexe de K-groupes de type multiplicatif. Alors l'accouplement (1) induit un isomorphisme entre le groupe discret $H^{1}(K,[\widehat{T} \rightarrow \widehat{S}])$ et le dual de Pontryagin $H_{\mathrm{fppf}}^{0}(K,[S \rightarrow T])^{D}$ de $H_{\mathrm{fppf}}^{0}(K,[S \rightarrow T])$.

Ici le dual de Pontryagin $A^{D}$ d'un groupe topologique abélien $A$ est le groupe des homomorphismes continus de $A$ dans le groupe discret $\mathbf{Q} / \mathbf{Z}$.

Démonstration. - On procède par dévissage en commençant par le cas où $T=0$. Comme $S$ est de type multiplicatif, on peut écrire une suite exacte

$$
0 \rightarrow S \rightarrow T_{1} \rightarrow T_{2} \rightarrow 0
$$

où $T_{1}$ et $T_{2}$ sont des tores. On obtient un diagramme commutatif à lignes exactes

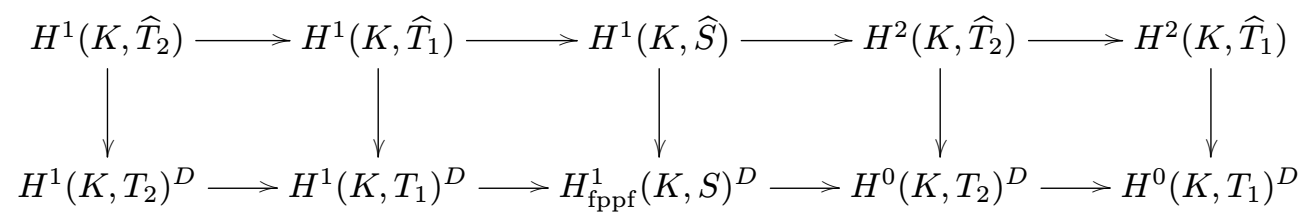

et le résultat découle alors du lemme des cinq joint au fait que pour chaque tore $T_{i}$ les flèches $H^{1}\left(K, \widehat{T}_{i}\right) \rightarrow H^{1}\left(K, T_{i}\right)^{D}$ et $H^{2}\left(K, \widehat{T}_{i}\right) \rightarrow H^{0}\left(K, T_{i}\right)^{D}$ induites par (1) sont des isomorphismes ([41], Th. III.6.9; noter que pour chaque tore $T_{i}$, le groupe $H^{1}\left(K, T_{i}\right)$ est fini tandis que $H^{0}\left(K, T_{i}\right)$ et son complété pour la topologie des sous-groupes ouverts d'indice fini ont même dual, [33], Lemme 2.2). 
Dans le cas général où $S$ et $T$ sont quelconques, on a, en posant $C^{\bullet}=[S \rightarrow T]$ et $\widehat{C}^{\bullet}=[\widehat{T} \rightarrow \widehat{S}]$, un diagramme commutatif

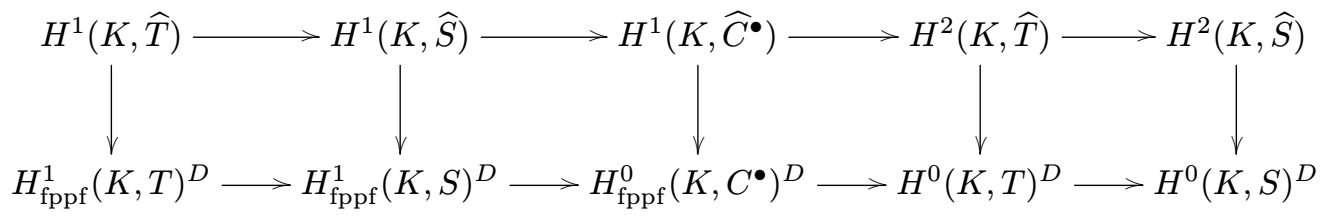

et le même argument de dévissage fonctionne à condition de savoir que pour un groupe de type multiplicatif $S$ quelconque, la flèche $H^{2}(K, \widehat{S}) \rightarrow H^{0}(K, S)^{D}$ est un isomorphisme. Or ceci est connu si $S$ n'a pas de $p$-torsion ([41], Cor. I.2.3, toujours avec le fait que $H^{0}(K, S)$ et son complété ont même dual), et le cas général s'en déduit immédiatement vu que si $\mu$ est un groupe de type multiplicatif de torsion $p$-primaire, on a $H_{\mathrm{fppf}}^{0}(K, \mu)=H^{2}(K, \hat{\mu})=0$ et $H^{1}(K, \hat{\mu}) \stackrel{\cong}{\longrightarrow} H_{\text {fppf }}^{1}(K, \mu)^{D}$ ([41], Prop. III.6.4 et Prop. III.6.10).

Remarque. - On pourrait montrer plus précisément qu'on obtient une dualité entre le groupe discret $H^{1}(K,[\widehat{T} \rightarrow \widehat{S}])$ et le complété (pour la topologie définie par les sous-groupes ouverts d'indice fini) de $H_{\mathrm{fppf}}^{0}(K,[S \rightarrow T])$; nous n'aurons pas besoin de ce résultat.

\section{Groupe de Brauer non ramifié, relation avec le complexe $K D^{\prime}(X)$}

Soit $p: X \rightarrow$ Spec $k$ une variété lisse et géométriquement intègre sur un corps $k$, dont on note $\operatorname{Br} X:=H^{2}\left(X, \mathbf{G}_{m}\right)$ le groupe de Brauer cohomologique. Rappelons que $X$ étant un schéma intègre régulier, la flèche canonique $\operatorname{Br} X \rightarrow \operatorname{Br} k(X)$ est injective (où $k(X)$ est le corps des fonctions de $X$ ) par le corollaire II.1.8 de [30]. Si $X^{c}$ est une compactification lisse de $X$, alors la flèche composée $\operatorname{Br} X^{c} \rightarrow \operatorname{Br} X \rightarrow \operatorname{Br} k(X)$ est injective, donc la flèche $\mathrm{Br} X^{c} \rightarrow \operatorname{Br} X$ est injective.

Comme dans [32], on considère le complexe de faisceaux étales sur Spec $k$ défini par

$$
K D(X):=\left(\tau_{\leq 1} \mathbf{R} p_{*} \mathbf{G}_{m, X}\right)[1]
$$

et on définit $K D^{\prime}(X)=(\operatorname{UPic} X)[1]$ comme le cône du morphisme canonique $\mathbf{G}_{m}[1] \rightarrow K D(X)$.

Posons $\operatorname{Br}_{1} X=\operatorname{ker}[\operatorname{Br} X \rightarrow \operatorname{Br} \bar{X}]$. Un argument de suite spectrale donne des flèches

$$
H^{1}\left(k, \mathbf{R} p_{*} \mathbf{G}_{m, X}\right) \rightarrow H_{\mathrm{fppf}}^{1}\left(k, \mathbf{R} p_{*} \mathbf{G}_{m, X}\right) \rightarrow \operatorname{Br} X=H^{2}\left(X, \mathbf{G}_{m}\right)=H_{\mathrm{fppf}}^{2}\left(X, \mathbf{G}_{m}\right)
$$

qui induisent un isomorphisme $H^{1}(k, K D(X)) \simeq \mathrm{Br}_{1} X$, et une flèche

$$
r: \mathrm{Br}_{1} X \simeq H^{1}(k, K D(X)) \rightarrow H^{1}\left(k, K D^{\prime}(X)\right)
$$

dont le noyau est $\operatorname{Br}_{0} X:=\operatorname{Im}[\operatorname{Br} k \rightarrow \operatorname{Br} X]$; de plus on a une suite exacte

$$
\mathrm{Br} k \rightarrow \mathrm{Br}_{1} X \stackrel{r}{\rightarrow} H^{1}\left(k, K D^{\prime}(X)\right) \rightarrow \operatorname{ker}\left[H^{3}\left(k, \mathbf{G}_{m}\right) \rightarrow H^{3}\left(X, \mathbf{G}_{m}\right)\right]
$$

([9], Prop. 2.18 et [32], page 7), ce qui fait que $r$ est de plus surjective si $H^{3}\left(k, \mathbf{G}_{m}\right)=0$ ou si $X(k) \neq \varnothing$. Si $m \in X(k)$ est un point rationnel de $X$, on notera $\operatorname{Br}_{1, m} X$ le sous-groupe de $\mathrm{Br}_{1} X$ constitué des éléments dont l'évaluation en $m$ est nulle. Ainsi $\mathrm{Br}_{1, m} X$ s'identifie à $\mathrm{Br}_{1} X / \mathrm{Br} k$. 
Proposition 4.1. - Soit $X$ une variété lisse, géométriquement intègre sur un corps $k$. On suppose que $X$ admet une compactification lisse $X^{c}$. Alors l'image réciproque du sous-groupe $\amalg_{\omega, \text { alg }}^{1}\left(K D^{\prime}(X)\right) \subset H^{1}\left(k, K D^{\prime}(X)\right)$ par $r$ est contenue dans l'image de $\operatorname{Br}_{1} X^{c}$ dans $\operatorname{Br}_{1} X$. Le même résultat vaut avec $\amalg_{\omega}^{1}\left(K D^{\prime}(X)\right)$ à la place de $\amalg_{\omega, \text { alg }}^{1}\left(K D^{\prime}(X)\right)$ si $k$ est un corps global.

Démonstration. - D'après [9], prop. 2.19, on a un diagramme commutatif exact

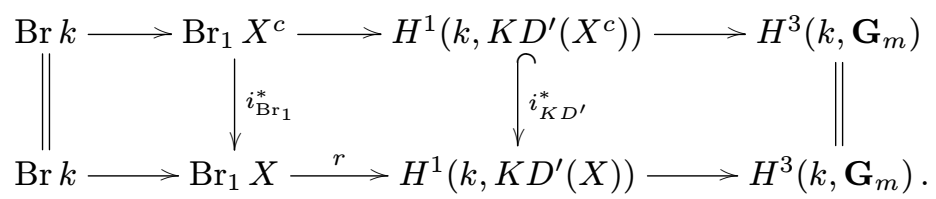

D'après [9], cor. 2.16, l'homomorphisme $i_{K D^{\prime}}^{*}$ est injectif et induit un isomorphisme

$$
\amalg_{\omega, \text { alg }}^{1}\left(K D^{\prime}\left(X^{c}\right)\right) \stackrel{\sim}{\longrightarrow} \amalg_{\omega, \text { alg }}^{1}\left(K D^{\prime}(X)\right) .
$$

Soit $\xi \in \operatorname{Br}_{1} X$ tel que $r(\xi) \in \amalg_{\omega, \text { alg }}^{1}\left(K D^{\prime}(X)\right)$, alors

$$
r(\xi) \in i_{K D^{\prime}}^{*}\left(\amalg_{\omega, \text { alg }}^{1}\left(K D^{\prime}\left(X^{c}\right)\right)\right) \subset \operatorname{Im} i_{K D^{\prime}}^{*},
$$

et par chasse au diagramme on obtient que $\xi \in \operatorname{Im} i_{\mathrm{Br}_{1}}^{*}$. L'argument avec $\amalg_{\omega}^{1}$ à la place de $\amalg_{\omega, \text { alg }}^{1}$ est identique.

Remarque. - Si $k$ est de caractéristique zéro, l'existence de $X^{c}$ est automatique par la résolution des singularités d'Hironaka. Dans ce cas $\operatorname{Br} X^{c}$ n'est autre que le groupe de Brauer non ramifié $\mathrm{Br}_{\mathrm{nr}} X$ de $X$ ([17], section 5). En caractéristique $p$, si $X^{c}$ existe, on sait juste que pour tout nombre premier $l$ différent de $p$, on a $\left(\mathrm{Br}_{\mathrm{nr}} X\right)\{l\}=\left(\mathrm{Br} X^{c}\right)\{l\}$ ([13], proposition 4.2.3).

Montrons maintenant une sorte de réciproque de la proposition 4.1, sur un corps global, sans supposer l'existence d'une compactification lisse de $X$.

Proposition 4.2. - Soient $k$ un corps global de caractéristique $p \geq 0, X$ une $k$-variété lisse et géométriquement intègre. Soit $\alpha \in\left(\mathrm{Br}_{\mathrm{nr}} X\right)\left\{p^{\prime}\right\}$ (resp. $\alpha \in \mathrm{Br} X^{c}$ si on suppose l'existence d'une compactification lisse $X^{c}$ de $\left.X\right)$. Alors pour presque toute place $v$ de $k$, $\alpha_{v} \in \operatorname{Br} X_{v}$ est orthogonal à $X\left(k_{v}\right)$ pour l'accouplement d'évaluation $X\left(k_{v}\right) \times \operatorname{Br} X_{v} \rightarrow$ $\mathrm{Br} k_{v} \cong \mathbf{Q} / \mathbf{Z}$, où on a noté $X_{v}:=X \times_{k} k_{v}$.

Démonstration. - Supposons tout d'abord l'existence d'une compactification lisse $X^{c}$ de $X$ sur $k$ (par exemple $k$ de caractéristique nulle). Il existe alors un ensemble fini de places $\Sigma$ de $k$ (contenant les éventuelles places archimédiennes) tel que la $k$-variété propre et lisse $X^{c}$ s'étende en un schéma propre et lisse $\chi^{c}$ au-dessus de l'anneau des $\Sigma$-entiers $\theta_{\Sigma}$. Soit $\alpha \in \operatorname{Br} X^{c}$. Quitte à augmenter $\Sigma$, on peut supposer que $\alpha$ est dans $\operatorname{Br} \chi^{c}$ car $\operatorname{Br} X^{c}$ est la limite inductive des $\operatorname{Br}\left(\chi^{c} \times{ }_{\vartheta_{\Sigma}} \vartheta_{\Sigma^{\prime}}\right)$ quand $\Sigma^{\prime}$ décrit la famille des ensembles finis de places de $k$ contenant $\Sigma$ (cf. [40], Lemme III.1.16). Or le groupe de Brauer de l'anneau des entiers $\theta_{v}$ de tout complété non archimédien $k_{v}$ de $k$ est nul; par conséquent on a $\alpha\left(P_{v}\right)=0$ pour toute $v \notin \Sigma$ et tout point local $P_{v} \in X\left(k_{v}\right)$ car par propreté $P_{v}$ s'étend en une $\theta_{v}$-section de $\chi^{c}$.

Traitons maintenant le cas général où $k$ est de caractéristique $p>0$, ce qui fait que l'existence d'une compactification lisse n'est pas connue. Soit $\alpha \in\left(\mathrm{Br}_{\mathrm{nr}} X\right)\left\{p^{\prime}\right\}$. Pour 
montrer la propriété voulue, on peut supposer que $\alpha \in\left(\mathrm{Br}_{\mathrm{nr}} X\right)\{l\}$, avec $l$ premier distinct de $p$. Le théorème de compactification de Nagata (cf. [19]) assure l'existence d'une immersion ouverte $X \rightarrow Z$, où $Z$ est une $k$-variété propre. Un théorème de Gabber (voir [36], exposé $\mathrm{X}$, théorème 2.1) assure qu'il existe une extension finie de corps $k^{\prime} / k$, de degré premier à $l$, et une $l^{\prime}$-altération $h: Y \rightarrow Z_{k^{\prime}}$, où $Y$ est une $k^{\prime}$-variété lisse. En particulier, le morphisme $h$ est propre, surjectif et génériquement fini de degré premier à $l$, et $Y$ est donc propre et lisse sur $k^{\prime}$. On considère alors le diagramme commutatif suivant, où les deux carrés sont des produits fibrés :

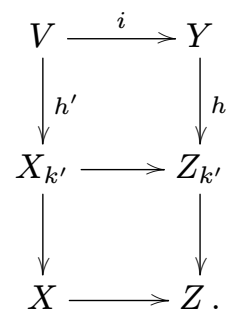

On note $\pi$ le morphisme composé $V \rightarrow X_{k^{\prime}} \rightarrow X$. Par fonctorialité du groupe de Brauer non ramifié (voir [17], lemme 5.5), on a $\pi^{*}(\alpha) \in\left(\operatorname{Br}_{\mathrm{nr}} V\right)\{l\}=(\operatorname{Br} Y)\{l\}$.

Le morphisme $\pi: V \rightarrow X$ étant génériquement fini de degré $d$ premier à $l$, il existe un ouvert affine $U$ de $X$ tel que la restriction $\pi_{U}: V_{U}:=V \times_{X} U \rightarrow U$ de ce morphisme soit un morphisme fini et plat sur $U$, donc dont le degré des fibres est un entier constant $d$ premier à $l$.

Soit $S$ un ensemble fini de places de $k$ tel que le diagramme (3) (ou plus exactement son analogue en remplaçant $X, X_{k^{\prime}}, V$ respectivement par $\left.U, U_{k^{\prime}}, V_{U}\right)$ s'étende en un diagramme de $\operatorname{Spec} \theta_{k, S}$-schémas

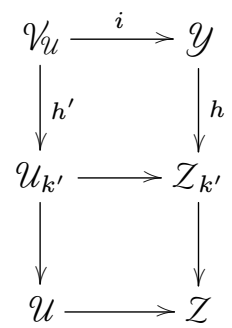

avec $\mathcal{Y}$ propre lisse sur Spec $\vartheta_{k^{\prime}, S^{\prime}}$, où $S^{\prime}$ désigne l'ensemble des places de $k^{\prime}$ au-dessus de $S$. Quitte à agrandir $S$, on peut aussi supposer que $\alpha \in \operatorname{Br} \mathcal{U}$ et que $\pi^{*}(\alpha) \in \operatorname{Br} \mathcal{Y}$ (car $\left.\pi^{*}(\alpha) \in \operatorname{Br} Y\right)$.

Soient $v \notin S$ et $x_{v} \in U\left(k_{v}\right)$. Puisque l'on a l'égalité $\left[k^{\prime}: k\right]=\sum_{w \mid v} e_{w} f_{w}$ (voir par exemple [43], théorème 7.6), le fait que $l$ ne divise pas $\left[k^{\prime}: k\right]$ assure qu'il existe une place $w$ de $k^{\prime}$ au-dessus de $v$ telle que $\left[k_{w}^{\prime}: k_{v}\right]=e_{w} f_{w}$ soit premier à $l$.

Puisque $\pi_{U}: V_{U} \rightarrow U_{k^{\prime}}$ est fini de degré premier à $l$, il existe une extension finie de corps $L / k_{w}^{\prime}$ et un point $y \in X(L)$ tels que $x_{v}=\pi_{U}(y)$, avec $\left[L: k_{w}^{\prime}\right]$ (et donc aussi $\left[L: k_{v}\right]$ ) premier à $l$.

4 e SÉRIE - TOME $46-2013-$ No $^{\circ}$ 
On a alors $\operatorname{Res}_{L / k_{v}}\left(\alpha\left(x_{v}\right)\right)=\left(\left(\pi_{U}\right)^{*}(\alpha)\right)(y)$ dans $\operatorname{Br} L$. Or on dispose du diagramme commutatif suivant :

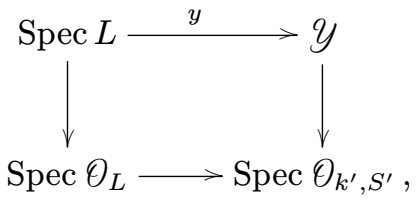

donc le critère valuatif de propreté assure l'existence d'une factorisation

$$
y: \operatorname{Spec} \theta_{L} \rightarrow \mathcal{Y} .
$$

Comme $\left(\pi_{U}\right)^{*}(\alpha) \in \operatorname{Br} \mathcal{Y}$, on en déduit que $\left(\left(\pi_{U}\right)^{*}(\alpha)\right)(y) \in \operatorname{Br} \theta_{L}=0$. Donc finalement, on a montré que $\operatorname{Res}_{L / k_{v}}\left(\alpha\left(x_{v}\right)\right)=0$ dans $\operatorname{Br} L$. Comme $\left[L: k_{v}\right]$ est premier à $l$ et que la restriction $\mathrm{Br} k_{v} \rightarrow \mathrm{Br} L$ correspond à la multiplication par $\left[L: k_{v}\right]$ (qui est premier à $l$ ) dans $\mathbf{Q} / \mathbf{Z}$, on obtient que $\alpha\left(x_{v}\right)=0$ dans $\operatorname{Br} k_{v}$. Par conséquent, l'élément $\alpha_{v} \in \operatorname{Br} X_{v}$ est orthogonal à $U\left(k_{v}\right)$ pour toute place $v \notin S$. Enfin, puisque $U$ est un ouvert non vide de la variété intègre $X$, le théorème des fonctions implicites donne que $U\left(k_{v}\right)$ est dense dans $X\left(k_{v}\right)$ pour la topologie $v$-adique. Or l'accouplement $\operatorname{Br} X_{v} \times X\left(k_{v}\right) \rightarrow \operatorname{Br} k_{v}$ est continu pour cette topologie, donc $\alpha_{v}$ est orthogonal à $X\left(k_{v}\right)$ tout entier, pour toute $v \notin S$. Cela conclut la preuve.

Voici une variante de la proposition précédente mettant en jeu des corps finis et locaux :

Proposition 4.3. - Soit $k$ un corps fini de caractéristique $p$. Soit $K=k((t))$. Soit $X$ une $k$-variété lisse et géométriquement intègre. Soit $\alpha \in\left(\mathrm{Br}_{\mathrm{nr}} X\right)\left\{p^{\prime}\right\}$. Alors pour tout $K$-point $P$ de $X$, on a $\alpha(P)=0$ dans $\operatorname{Br} K$.

Démonstration. - Supposons d'abord que $X$ admette une compactification lisse $X^{c}$. On a alors $\alpha \in \operatorname{Br} X^{c}$. Soit $\alpha_{K}$ l'image de $\alpha$ dans $\operatorname{Br} X_{K}$. Alors $\alpha_{K}$ provient de $\operatorname{Br}\left(X_{\vartheta_{K}}^{c}\right)$, où $\theta_{K}=k[[t]]$, donc pour tout $K$-point $P$ de $X$ (qui s'étend par propreté en une $\theta_{K}$-section de $\left.X^{c} \rightarrow \operatorname{Spec} \theta_{K}\right)$ on a $\alpha_{K}(P) \in \operatorname{Br} \theta_{K}=0$ d'où $\alpha(P)=0$.

On se ramène enfin au cas où on a une compactification lisse par le même argument (utilisant le théorème de Gabber) que dans la proposition 4.2.

\section{Le groupe de Brauer géométrique}

Dans cette section, on montre que dans le cas des espaces homogènes à stabilisateurs connexes sur un corps algébriquement clos, le groupe de Brauer d'un modèle projectif lisse est trivial. Plus précisément le théorème principal de cette section est le suivant :

THEORÈme 5.1. - Soit $k$ un corps séparablement clos.

Soit $G$ un k-groupe linéaire connexe lisse et soit $H$ un k-sous-groupe connexe lisse de $G$. On note $X:=G / H$ et on désigne par $X^{c}$ une compactification lisse de $X$. On suppose l'une des conditions suivantes

- $k$ est de caractéristique nulle.

- $k$ est algébriquement clos (i.e. $k$ est parfait) et $H$ est produit semi-direct de $H^{\mathrm{u}}$ par $H^{\mathrm{red}}$.

- H et $G$ sont réductifs. 
Alors $\left(\operatorname{Br} X^{c}\right)\{l\}=0$, pour tout l premier à la caractéristique de $k$.

Remarque. - Ce résultat étend le résultat classique de Bogomolov ([1], théorème 2.4, voir aussi [17], théorème 9.13), qui concerne le cas où $k=\mathbf{C}$ et $G$ est semi-simple simplement connexe. Cela répond en particulier par l'affirmative à la remarque 9.14 de [17], ainsi qu'à la question qui suit le théorème 1.4 de [16], où les auteurs demandent si le résultat de Bogomolov s'étend (en caractéristique nulle) au cas où $G$ est un groupe linéaire connexe quelconque.

D’autre part, quelques mois après que le présent article a été terminé, Borovoi ([6]) a donné une preuve simple du théorème 5.1 en caractéristique zéro en s'appuyant sur le résultat de Bogomolov (tandis que la démonstration que nous allons donner ici est indépendante).

On commence par un lemme général (on remercie J.-L. Colliot-Thélène pour la démonstration qui suit) :

Lemme 5.2. - Soit $X$ une variété lisse géométriquement intègre sur un corps $k$. Soit $\alpha \in \operatorname{Br} X$. On suppose qu'il existe une extension de corps $K$ de $k$ telle que la restriction $\alpha_{K} \in \operatorname{Br} X_{K}$ soit nulle.

Supposons $k$ parfait ou $\alpha \in(\operatorname{Br} X)\left\{p^{\prime}\right\}$.

Alors $\alpha$ est algébrique (i.e. la restriction de $\alpha$ à $\mathrm{Br} \bar{X}$ est nulle).

Démonstration. - Puisque $\operatorname{Br} X_{K}$ est la limite inductive des groupes $\operatorname{Br} X_{A}$, où $A$ décrit les $k$-algèbres de type fini contenues dans $K$, il existe une $k$-algèbre de type fini $A_{0}$ contenue dans $K$ telle que $\alpha_{A_{0}}=0 \in \operatorname{Br} X_{A_{0}}$. Fixons un point fermé $s \in \operatorname{Spec} A_{0}$. Par fonctorialité, on a $\alpha_{k(s)}=0 \in \operatorname{Br} X_{k(s)}$, et par construction $k(s)$ est une extension finie de $k$, donc $\alpha_{k^{\text {alg }}}=0 \in \operatorname{Br} X_{k^{\text {alg }}}$, où $k^{\text {alg }}$ est une clôture algébrique de $k$ contenant $\bar{k}$. Cela termine la preuve si $k$ est parfait. Sinon, montrons que le morphisme naturel $(\operatorname{Br} \bar{X})\left\{p^{\prime}\right\} \rightarrow$ $\left(\operatorname{Br} X_{k^{a l g}}\right)\left\{p^{\prime}\right\}$ est injectif : puisque l'extension $k^{\text {alg }}(X) / \bar{k}(X)$ est purement inséparable, on sait que pour tout $n$ premier à $p$, le morphisme $H^{2}\left(\bar{k}(X), \mu_{n}\right) \rightarrow H^{2}\left(k^{\mathrm{alg}}(X), \mu_{n}\right)$ est injectif, donc cela assure que $(\operatorname{Br} \bar{k}(X))\left\{p^{\prime}\right\} \rightarrow\left(\operatorname{Br} k^{\text {alg }}(X)\right)\left\{p^{\prime}\right\}$ est injectif, donc $(\operatorname{Br} \bar{X})\left\{p^{\prime}\right\} \rightarrow\left(\operatorname{Br} X_{k^{\text {alg }}}\right)\left\{p^{\prime}\right\}$ aussi. On conclut finalement que $\alpha_{\bar{k}}=0$ dans $\operatorname{Br} \bar{X}$.

Preuve du théorème 5.1. - La preuve de ce résultat se fait en plusieurs étapes.

Étape 1. - Cas de la clôture séparable d'un corps global.

Lemme 5.3. - Soit $k$ un corps global. Soit l premier à $\operatorname{Car}(k)$.

- Soient $H$ et $G$ deux $k$-groupes réductifs, avec $H \subset G$. On pose $X:=G / H$. Soit $X^{c}$ une compactification lisse de $X$.

Alors $\left(\mathrm{Br} X^{c}\right)\{l\}=\left(\mathrm{Br}_{1} X^{c}\right)\{l\}$.

- Si H, G, X et $X^{c}$ sont définis sur $\bar{k}$, alors $\left(\operatorname{Br} X^{c}\right)\{l\}=0$.

4 e SÉRIE - TOME $46-2013$ - No 4 
Remarque. - Grâce à la proposition 4.2 et au lemme 5.2, ce lemme reste valable sans supposer l'existence de la compactification lisse $X^{c}$ de $X$, en remplaçant $\operatorname{Br} X^{c} \operatorname{par}^{B} r_{n r} X$. Noter aussi qu'en particulier $H$ et $G$ sont supposés lisses.

Démonstration. - Tout d'abord, il est clair que le second point du lemme est une conséquence du premier. En effet, si tout est défini sur $\bar{k}$, et si $\alpha \in\left(\operatorname{Br} X^{c}\right)\{l\}$, alors il existe une extension finie séparable $K / k$ telle que $H, G, X, X^{c}$ et $\alpha$ soient définis sur $K$. Or $K$ est un corps global, donc $\alpha$ vu dans le groupe de Brauer de la $K$-variété $X^{c}$ est algébrique par le premier point, donc $\alpha$ est nul dans le groupe de Brauer de la $\bar{k}$-variété $X^{c}$.

Montrons maintenant le premier point. D'après [16], lemme 1.5, on peut supposer que le groupe $G$ est quasi-trivial. Soit $\alpha \in\left(\mathrm{Br}_{e} X^{c}\right)\{l\}$. On souhaite montrer que $\alpha$ est algébrique, c'est-à-dire que sa restriction à $\mathrm{Br} \bar{X}^{c}$ est nulle.

On note $\pi: G \rightarrow X$ le morphisme quotient.

Quitte à remplacer $k$ par une extension finie séparable, on peut supposer que les groupes réductifs $H$ et $G$ sont $k$-déployés.

Alors $\pi^{*}(\alpha)=0$ dans $\mathrm{Br}_{\mathrm{nr}}{ }_{e} G$ (puisque $\left(\mathrm{Br}_{\mathrm{nr}} G\right)\{l\}=(\mathrm{Br} k)\{l\}$, vu que $G$ est réductif déployé, donc rationnel : voir [2], corollaires 14.14 et 18.8).

Par conséquent, la suite exacte du théorème 2.4 de [8] (valable en caractéristique positive, avec la même preuve, en supposant $H$ et $G$ réductifs) assure qu'il existe $\beta \in$ Pic $H$ tel que $\delta(\beta)=\alpha$, où $\delta: \operatorname{Pic~} H \rightarrow \operatorname{Br} X$ est le morphisme défini par Colliot-Thélène et $\mathrm{Xu}$ ([18], section 2, p.314).

Puisque $\alpha \in \operatorname{Br} X$ est non ramifié (i.e. appartient à $\mathrm{Br} X^{c}$ ) et que le groupe de Brauer de l'anneau des entiers $\theta_{v}$ est nul, on obtient que pour presque toute place $v$ de $k$, la localisation $\alpha_{v} \in \operatorname{Br} X_{v}:=\operatorname{Br}\left(X \times_{k} k_{v}\right)$ est orthogonale à $X\left(k_{v}\right)$, pour l'accouplement canonique d'évaluation (voir proposition 4.2).

On considère le diagramme commutatif suivant :

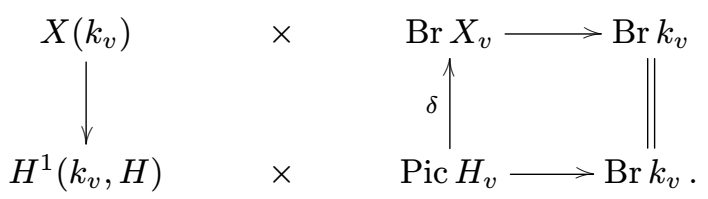

Dans ce diagramme, le premier accouplement horizontal est l'accouplement d'évaluation usuel. Pour la définition de la seconde ligne et la commutativité du diagramme, voir [18], proposition 2.9.

Or $G$ est quasi-trivial, donc $H^{1}\left(k_{v}, G\right)=1$ pour toute place finie $v$ de $k$. Donc l'application caractéristique $X\left(k_{v}\right) \rightarrow H^{1}\left(k_{v}, H\right)$ est surjective. Donc le diagramme (4) assure que, pour presque toute place $v, \beta_{v} \in \operatorname{Pic} H_{v}$ est orthogonal à $H^{1}\left(k_{v}, H\right)$.

Soit $T_{H}$ un tore maximal de $H$ et $T_{H^{\text {sc }}}$ son image réciproque par le morphisme canonique $H^{\text {sc }} \rightarrow H$. Notons $C_{H}:=\left[T_{H^{\mathrm{sc}}} \rightarrow T_{H}\right]$ et $\widehat{C}_{H}:=\left[\widehat{T}_{H} \rightarrow \widehat{T}_{H^{\mathrm{sc}}}\right]$. Rappelons qu'on dispose alors du groupe $H_{\mathrm{ab}}^{1}\left(k_{v}, H\right):=\mathbf{H}^{1}\left(k_{v}, C_{H}\right)$ et d'un morphisme d'abélianisation $\mathrm{ab}_{H}^{1}: H^{1}\left(k_{v}, H\right) \rightarrow H_{\mathrm{ab}}^{1}\left(k_{v}, H\right)$ qui est surjectif pour toute place $v$ de $k$ ([5], théorème 5.4 en caractéristique zéro. En caractéristique positive, le théorème 5.5 et l'exemple 5.4(i) de [28] assurent le résultat). 
On a un diagramme commutatif :

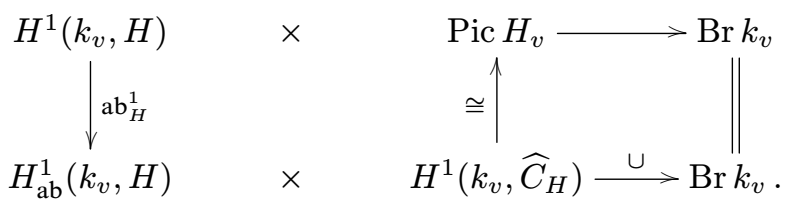

Or le morphisme $\mathbf{H}^{1}\left(k_{v}, \widehat{C}_{H}\right) \rightarrow$ Pic $H_{v}$ est un isomorphisme (voir [9], corollaire 5) et l'application $\mathrm{ab}_{H}^{1}$ est surjective, donc l'image $q_{v}$ de $\beta_{v}$ dans $\mathbf{H}^{1}\left(k_{v}, \widehat{C}_{H}\right)$ est orthogonale à $H_{\mathrm{ab}}^{1}\left(k_{v}, H\right)$ pour le cup-produit. Or l'accouplement $H_{\mathrm{ab}}^{1}\left(k_{v}, H\right) \times \mathbf{H}^{1}\left(k_{v}, \widehat{C}_{H}\right) \rightarrow \mathrm{Br} k_{v}$ est une dualité parfaite de groupes finis (voir [22], théorème 3.1 pour la caractéristique nulle; la preuve en caractéristique positive est similaire), donc $q_{v}=0$, donc $\beta_{v}=0$.

Finalement, on a montré que $\beta_{v}=0$ pour presque toute place $v$, donc on a aussi $\alpha_{v}=0$ pour presque toute place $v$. Ceci implique en particulier que $\alpha$ est algébrique via le lemme 5.2.

Remarque. - Sur un corps $p$-adique, la dualité entre $H^{1}\left(k_{v}, H\right)$ et Pic $H_{v}$ (variante de résultats de Kottwitz) avait déjà été obtenue par Colliot-Thélène ([14], théorème 9.1) en utilisant les résolutions flasques.

Étape 2. - Cas d'un corps algébriquement clos de caractéristique nulle.

Dans cette partie, on démontre le théorème 5.1 dans le cas où $k$ est un corps algébriquement clos de caractéristique nulle.

On suppose d'abord que $H$ et $G$ sont réductifs. On voit $\overline{\mathbf{Q}}$ comme un sous-corps de $k$. La théorie des groupes réductifs assure qu'il existe des $\overline{\mathbf{Q}}$-groupes réductifs $H^{\prime}$ et $G^{\prime}$ tels que $H_{k}^{\prime}=H$ et $G_{k}^{\prime}=G$. Par un théorème de Vinberg rédigé par Margaux (voir théorème 1.1 de [39]), appliqué à l'inclusion (définie sur $k$ ) $i: H \rightarrow G$, il existe une inclusion $i^{\prime}: H^{\prime} \rightarrow G^{\prime}$ définie sur $\overline{\mathbf{Q}}$ et $g \in G(k)$ tels que $i=\operatorname{int}(g) \circ i_{k}^{\prime}$. On dispose alors d'une $\overline{\mathbf{Q}}$-variété $X^{\prime}:=G^{\prime} / H^{\prime}$.

Le $k$-morphisme $\operatorname{int}(g): G \rightarrow G$ induit alors un $k$-isomorphisme $\varphi: X_{k}^{\prime} \rightarrow X$. On en déduit donc un isomorphisme de groupes $\mathrm{Br} X^{c} \cong \mathrm{Br} X_{k}^{\prime c}$.

Or la rigidité de la cohomologie non ramifiée à coefficients finis assure que $\operatorname{Br}{X^{\prime}}^{c}=\operatorname{Br}{X_{k}^{\prime c}}_{k}^{c}$ ([13], théorème 4.4.1).

Le lemme 5.3 de l'étape 1 assure que $\operatorname{Br} X^{\prime c}=0$, d'où finalement $\operatorname{Br} X^{c}=0$.

Supposons maintenant seulement $H$ réductif. On définit $Y:=X / G^{\mathrm{u}}=G /\left(H . G^{\mathrm{u}}\right)$. Alors $Y$ est un espace homogène de $G^{\text {red }}$ à stabilisateur réductif, donc par la preuve précédente, on a $\operatorname{Br} Y^{c}=0$ si $Y^{c}$ est une compactification lisse de $Y$. D'autre part la fibre générique du morphisme $X \rightarrow Y$ est un espace homogène $W$ de $G^{u}$, elle admet donc un point rationnel ([4], Lemme 3.2) sur le corps des fonctions $K$ de $Y$. En particulier on a un $K$-morphisme $G_{K}^{u} \rightarrow W$ qui est un torseur sous un $K$-groupe unipotent, donc un torseur trivial. Ceci implique que $W$ est $K$-rationnel (puisque c'est le cas de tout groupe unipotent en caractéristique zéro). Finalement la variété $X$ est stablement $k$-birationnelle à $Y$, donc $\operatorname{Br} X^{c} \cong \operatorname{Br} Y^{c}$, d'où $\operatorname{Br} X^{c}=0$.

On ne suppose plus maintenant ni $H$, ni $G$ réductifs. Le théorème de Mostow assure l'existence d'une décomposition en produit semi-direct $H=H^{\mathrm{u}} \rtimes H^{\text {red }}$. On considère 
alors le quotient $Z:=G / H^{\text {red }}$. On dispose d'un morphisme naturel $Z \rightarrow X$. Par [24], lemme 2.7, toute fibre (en particulier la fibre générique) de ce morphisme $Z \rightarrow X$ a un point rationnel. On conclut alors comme ci-dessus que $Z$ est stablement $k$-birationnelle à $X$, d'où $\operatorname{Br} X^{c} \cong \operatorname{Br} Z^{c}$. Or $\operatorname{Br} Z^{c}=0$ par le cas précédent ( $Z$ est un espace homogène de $G$ à stabilisateur réductif $H^{\text {red }}$ ), donc $\operatorname{Br} X^{c}=0$, ce qui conclut la preuve du théorème 5.1 dans le cas de caractéristique nulle.

Étape 3. - Cas d'un corps séparablement clos de caractéristique positive.

En caractéristique positive, le théorème de Vinberg-Margaux n'est pas vérifié pour des groupes réductifs semi-simples : il vaut pour les groupes linéairement réductifs (voir [39], remarque 5.2 pour un contre-exemple). Par conséquent, on ne peut pas adapter la méthode de l'étape 2 pour déduire le théorème du cas des corps globaux. On va donc utiliser une autre méthode, consistant à se ramener aux corps finis.

Notons d'abord qu'on peut supposer $G$ et $H$ réductifs, car sinon les hypothèses supplémentaires ( $k$ parfait et $H$ produit semi-direct de $H^{u}$ par $H^{\text {red }}$ ) permettent de se ramener à cette situation comme à la fin de l'étape 2 .

On commence par les corps finis :

Lemme 5.4. - Si $k$ est la clôture algébrique d'un corps fini $\mathbf{F}$, alors on a $\left(\operatorname{Br}_{\mathrm{nr}} X\right)\{l\}=0$, pour tout $l \neq \operatorname{Car}(\mathbf{F})$.

Démonstration. - On considère l'extension de corps $k \subset K:=\overline{\mathbf{F}(t)}$. Par le lemme 5.3 et la remarque qui le suit, on sait que $\left(\mathrm{Br}_{\mathrm{nr}} X_{K}\right)\{l\}=0$. Alors le lemme 5.2 assure que tous les éléments de $\left(\mathrm{Br}_{\mathrm{nr}} X\right)\{l\}$ sont algébriques. Or $k$ est séparablement clos, donc $\left(\mathrm{Br}_{\mathrm{nr}} X\right)\{l\}=0$.

Démontrons maintenant le théorème 5.1 dans le cas général, où $k$ est un corps séparablement clos de caractéristique $p>0$. Soit $\alpha \in\left(\operatorname{Br} X^{c}\right)\{l\}$. Alors il existe une sous-extension de type fini $L / \mathbf{F}_{p}$ de $k / \mathbf{F}_{p}$, telle que $H, G, X$ et $\alpha$ soient définis sur $L$.

En utilisant la suite exacte

$$
0 \rightarrow \operatorname{Pic} H_{K} \stackrel{\delta}{\rightarrow} \operatorname{Br} X_{K} \stackrel{\pi^{*}}{\rightarrow} \operatorname{Br} G_{K}
$$

pour toute extension $K / L$, ainsi que le fait que $\operatorname{Br}_{n r}\left(G_{\bar{L}}\right)\{l\}=0$ (puisque $G_{\bar{L}}$ est rationnel car déployé), on sait qu'il existe une extension finie séparable $K / L$ et $p \in \operatorname{Pic} H_{K}$ tels que l'image $\alpha_{K}$ de $\alpha$ dans $\operatorname{Br} X_{K}$ vérifie $\alpha_{K}=\delta(p)$, avec de plus la propriété que $H^{\text {tor }}$ soit $K$-déployé. On peut en outre supposer que le groupe fondamental $\mu_{H}$ de $H^{\text {ss }}$ est déployé par $K$.

Pour montrer le théorème, il suffit de montrer que $\alpha_{K}=0$.

Puisque Pic $H_{K}^{\text {tor }}=0$, le morphisme Pic $H_{K} \rightarrow$ Pic $H_{K}^{\text {ss }}$ est injectif.

On sait que $p$ est représentable par une extension centrale de $K$-groupes algébriques

$$
1 \rightarrow \mathbf{G}_{m K} \rightarrow H_{0} \rightarrow H_{K} \rightarrow 1 .
$$

On note $H_{1}$ le pull-back de cette extension par $H_{K}^{\text {ss }} \rightarrow H_{K}$.

La trivialité de Pic $H_{K}^{\text {sc }}$ assure que le pull-back de cette suite exacte par $H_{K}^{\text {sc }} \rightarrow H_{K}^{\text {ss }}$ est une suite scindée. 
Il existe alors une $\mathbf{F}_{p}$-algèbre intègre de type fini $B$ de corps des fractions $K$, des $B$-schémas en groupes $\mathcal{H}, \mathscr{G}, \mathcal{H}^{\mathrm{ss}}$, deux $B$-schémas $\chi$ et $\chi^{c}$ tels que

- les fibres génériques de $\mathcal{H}, \mathscr{G}, \mathcal{H}^{\mathrm{ss}}, \chi$ et $\chi^{c}$ sont respectivement $H_{K}, G_{K}, H_{K}^{\mathrm{ss}}, X_{K}$ et $X_{K}^{c}$.

- $\mathscr{G}$ est un $B$-schéma en groupes quasi-trivial (extension d'un tore quasi-trivial par un schéma en groupes semi-simple simplement connexe; en particulier le groupe de Picard des fibres géométriques de $\mathscr{G}$ est nul).

- $\mathscr{H}$ est un $B$-schéma en groupes réductif, et on a une suite exacte centrale de $B$-schémas en groupes

$$
1 \rightarrow \mu_{H, B} \rightarrow \mathcal{H}^{\mathrm{sc}} \rightarrow \mathcal{H}^{\mathrm{ss}} \rightarrow 1
$$

avec $\mathcal{H}^{\mathrm{sc}}$ semi-simple simplement connexe et $\mu_{H, B}$ fini de type multiplicatif et déployé.

- la suite (5) s'étend en une suite exacte centrale sur $B$

$$
1 \rightarrow \mathbf{G}_{m B} \rightarrow \mathcal{H}_{0} \rightarrow \mathcal{H} \rightarrow 1
$$

dont la classe $p_{B}$ dans Pic $\mathcal{H}$ a pour fibre générique $p \in \operatorname{Pic} H_{K}$. On peut de même étendre l'extension associée à $H_{1}$, ce qui donne une classe $p_{B}^{\prime} \in \operatorname{Pic} \mathcal{H}^{\text {ss }}$ (image de $p_{B}$ ), dont la fibre générique $p^{\prime}$ est l'image de $p$ dans Pic $H_{K}^{\text {ss }}$. On peut également supposer que la suite déduite par pull-back via $\mathscr{H}^{\text {sc }} \rightarrow \mathscr{H}^{\text {ss }}$ est scindée, ce qui implique que l'image de $p_{B}^{\prime}$ dans Pic $\mathcal{H}^{\text {sc }}$ est nulle.

- $H_{K} \rightarrow G_{K}$ s'étend en un sous-schéma en groupes fermé $\mathcal{H} \rightarrow \mathscr{G}$ et $\chi$ s'identifie au quotient $\mathscr{G} / \mathcal{H}$.

- $x \in X(k)$ s'étend en $x \in \chi(B)$.

- $\chi^{c}$ est un $B$-schéma projectif et lisse.

- $X \rightarrow X^{c}$ se prolonge en $\chi \rightarrow \chi^{c}$.

- pour tout $s \in \operatorname{Spec} B$, la fibre $\chi_{s}^{c}$ est une compactification lisse de $\chi_{s}$ sur $k(s)$.

- la classe $\alpha_{K} \in \operatorname{Br} X_{K}^{c}$ s'étend en une classe $A \in \operatorname{Br} \chi^{c}$, qui coïncide avec l'image de l'extension $\mathscr{H}_{0}$ (vue dans $\operatorname{Pic} \mathscr{H}$ ) via le morphisme $\operatorname{Pic} \mathscr{H} \rightarrow \operatorname{Br} \chi$ (construit comme dans le cas des corps, via le $\mathcal{H}$-torseur $\mathscr{G} \rightarrow \chi$ et la suite exacte $1 \rightarrow \mathbf{G}_{m B} \rightarrow \mathscr{H}_{0} \rightarrow$ $\mathcal{H} \rightarrow 1)$.

Fixons maintenant un point fermé $s \in \operatorname{Spec} B$. Par construction, le corps résiduel de $s$, noté $k(s)$, est un corps fini.

Les propriétés des modèles qu'on a considérés assurent que la $k(s)$-compactification lisse $\chi_{s} \rightarrow \chi_{s}^{c}$ vérifie les hypothèses du lemme 5.4. La classe $A_{s} \in \mathrm{Br} \chi_{s}^{c}$ est l'image de la classe $p_{s}$ de $\mathscr{H}_{0 s}$ (vue dans $\operatorname{Pic} \mathcal{H}_{s}$ ) par le morphisme $\delta_{s}: \operatorname{Pic} \mathcal{H}_{s} \rightarrow \operatorname{Br} \chi_{s}$. Notons $p_{s}^{\prime}$ l'image de $p_{s}$ dans Pic $\mathcal{H}_{s}^{\text {ss }}$, alors $p_{s}^{\prime}$ est aussi la fibre en $s$ de $p_{B}^{\prime} \in \operatorname{Pic} \mathcal{H}^{\text {ss }}$. Comme Pic $\bar{H}_{s}$ s'injecte dans $\operatorname{Br} \bar{X}_{s}$ (car Pic $\bar{G}_{s}=0$ ) et $A_{s}$ est algébrique (lemme 5.4), on obtient que l'image de $p_{s}$ dans Pic $\bar{H}_{s}$ est nulle. Mais Pic $H_{s}^{\text {ss }}$ s'injecte dans Pic $\bar{H}_{s}^{\text {ss }}$ puisque $H_{s}^{\text {ss }}$ est semi-simple; on en déduit que $p_{s}^{\prime}=0$.

Or on dispose d'une suite exacte de groupes

$$
0 \rightarrow H^{0}\left(B, \widehat{\mu_{H, B}}\right) \rightarrow \operatorname{Ext}_{B}^{c}\left(\mathcal{H}^{\mathrm{ss}}, \mathbf{G}_{m_{B}}\right) \rightarrow \operatorname{Ext}_{B}^{c}\left(\mathcal{H}^{\mathrm{sc}}, \mathbf{G}_{m_{B}}\right)
$$

où $\operatorname{Ext}_{B}^{c}(\ldots)$ désigne le groupe abélien des classes d'extensions centrales de $B$-faisceaux en groupes (voir [18], p.313). 
Comme l'image de $p_{B}^{\prime}$ dans Pic $\mathcal{H}^{\mathrm{sc}}$ est nulle, la classe $p_{B}^{\prime}$ provient d'un élément $\beta \in H^{0}\left(B, \widehat{\mu_{H, B}}\right)$, dont l'image $\beta_{s}$ dans $H^{0}\left(k(s), \widehat{\mu_{H, s}}\right)$ est nulle (car $\left.p_{s}^{\prime}=0\right)$. Or $\widehat{\mu_{H, B}}$ est un $B$-groupe constant car $\mu_{H, B}$ est fini de type multiplicatif déployé ; ceci implique que le morphisme

$$
H^{0}\left(B, \widehat{\mu_{H, B}}\right) \rightarrow H^{0}\left(k(s), \widehat{\mu_{H, s}}\right)
$$

est injectif. Alors $\beta_{s}=0$ implique que $\beta=0$ dans $H^{0}\left(B, \widehat{\mu_{H, B}}\right)$ ce qui implique $p_{B}^{\prime}=0$. Ainsi la fibre générique $p^{\prime}$ de $p_{B}^{\prime}$ est nulle dans Pic $H_{K}^{\text {ss }}$. Enfin, le morphisme Pic $H_{K} \rightarrow \operatorname{Pic} H_{K}^{\text {ss }}$ est injectif, d'où $p=0$ dans Pic $H_{K}$, donc $\alpha_{K}=0$ dans $\operatorname{Br} X_{K}$, ce qui achève la preuve du théorème 5.1 .

\section{Une formule de compatibilité}

On considère un $k$-groupe lisse et connexe $G$ (supposé réductif pour $k$ non parfait) et un $k$-sous-groupe algébrique $H$ de $G$. Rappelons qu'alors le $k$-groupe semi-simple $G^{\text {ss }}$ est simplement connexe si et seulement si $\operatorname{Pic} \bar{G}=0$ via [44], lemme 6.9(iii) et remarque 6.11.3 joints au fait que le noyau du revêtement universel $G^{\text {sc }} \rightarrow G^{\text {ss }}$ est un $k$-groupe fini de type multiplicatif [34].

DÉfinition 6.1. - Soit $H$ un $k$-groupe algébrique. On dit que $H$ est sans caractères si le seul morphisme de $\bar{k}$-groupes de $\bar{H}$ dans $\mathbf{G}_{m}$ est le morphisme constant. On dit que $H$ est $d e$ type (ssumult) s'il existe une suite exacte de $k$-groupes

$$
1 \rightarrow L \rightarrow H \rightarrow S \rightarrow 1
$$

vérifiant : $S$ est lisse de type multiplicatif et $L$ est lisse, connexe, sans caractères.

Noter en particulier qu'un groupe de type (ssumult) est lisse. Si $k$ est de caractéristique zéro, tout $k$-groupe connexe ou encore tout $k$-groupe commutatif est de type (ssumult). Si $k$ est parfait de caractéristique $p>0$, tout $k$-groupe lisse et connexe, ou encore tout $k$-groupe lisse commutatif avec $H / H^{0}$ sans $p$-torsion, est de type (ssumult); en effet ceci résulte de la structure des groupes algébriques sur un corps parfait. Plus généralement, si $k$ est parfait, un $k$-groupe lisse $H$ est de type (ssumult) si et seulement s'il vérifie la condition de [7], section 3 que le noyau de $H \rightarrow H^{\text {mult }}$ est connexe sans caractères, où $H^{\text {mult }}$ est le quotient maximal de $H$ qui est de type multiplicatif. La situation est plus compliquée sur un corps imparfait, parce qu'on n'a pas en général de radical unipotent défini sur $k$.

Soit $X:=G / H$ un espace homogène de $G$ possédant un $k$-point. D'après [27], Prop. III.3.1.1, on a pour tout $k$-schéma $Z$ une bijection fonctorielle $\tau_{Z}: X(Z) \rightarrow H^{0}(Z,[H \rightarrow G])$ qui induit par changement de base, pour tout $k$-point $s$ de $Z$, une bijection $X(k) \rightarrow$ $H^{0}(k,[H \rightarrow G])$. Pour $Z=X$, la classe $\tau_{X}$ (id) correspondant à l'identité de $X$ donne une classe $[[X]] \in H_{\mathrm{fppf}}^{0}(X,[H \rightarrow G])$ induite par la trivialisation du $X$-torseur $G \wedge^{H} G$ donnée par le neutre $e \in G(k)$. Ici $G \wedge^{H} G$ est le torseur sous $G$ obtenu par produit contracté du $X$-torseur sous $H$ donné par $G \rightarrow X$, cf. [48], lemme 2.2.3. On obtient ainsi une bijection

$$
\tau: X(k) \rightarrow H_{\mathrm{fppf}}^{0}(k,[H \rightarrow G]) \quad x \mapsto[[X]](x)
$$

induite par l'évaluation. 
THEORÈme 6.2. - Soit G un k-groupe lisse et connexe, réductif pour k non parfait, vérifiant $\operatorname{Pic} \bar{G}=0$. On pose $T=G^{\text {tor }}$. Soit $H$ un k-sous-groupe lisse de $G$, supposé de composante neutre réductive ou de type (ssumult) pour $k$ non parfait; on note $S=H^{\text {mult }}$ le quotient maximal de $H$ qui est de type multiplicatif. Soit $X=G / H$. Alors :

(a) Il existe un isomorphisme $K D^{\prime}(X) \simeq[\widehat{T} \rightarrow \widehat{S}]$ dans $\mathscr{D}(k)$ qui induit un isomorphisme fonctoriel

$$
\Phi_{X}: \mathrm{Br}_{1, e} X \rightarrow H^{1}(k,[\widehat{T} \rightarrow \widehat{S}])
$$

(b) Notons

$$
\mathrm{ab}^{0}=(\mathrm{ab} \circ \tau): X(k) \rightarrow H_{\mathrm{fppf}}^{0}(k,[S \rightarrow T])
$$

la composée de $\tau$ avec l'application d'abélianisation

$$
\mathrm{ab}: H_{\mathrm{fppf}}^{0}(k,[H \rightarrow G]) \rightarrow H_{\mathrm{fppf}}^{0}(k,[S \rightarrow T]) .
$$

Alors on a la formule :

$$
\Phi_{X}(\alpha) \cup \mathrm{ab}^{0}(x)=-\alpha(x)
$$

pour tout $x \in X(k)$ et tout $\alpha \in \mathrm{Br}_{1, e} X$, où $\cup$ est l'accouplement (1).

Remarque. - Nous aurons notamment à appliquer le théorème sur un corps local de caractéristique $p>0$, imparfait donc. L'hypothèse faite sur $H$ implique alors que $S=H^{\text {mult }}$ est bien défini. Noter aussi qu'avec les hypothèses de lissité faites, on peut remplacer ici les $H_{\mathrm{fppf}}^{0}$ par des $H^{0}$ étales.

Démonstration. - (a) Ceci a été établi par le premier auteur et van Hamel [10] en caractéristique zéro. La même preuve fonctionne en caractéristique quelconque une fois qu'on a fait les hypothèses supplémentaires sur $H$ du théorème 6.2.

(b) La démonstration de cette compatibilité va occuper le reste de cette section. Voir également l'appendice $\mathrm{B}$ pour une démonstration indépendante du théorème 6.2 quand $H$ est de type (ssumult). On pourra également se reporter au lemme 4.5.1 de [20] pour une preuve de la compatibilité analogue à celle que nous donnons maintenant.

Définition 6.3. - Une paire de k-groupes est une paire $(G, H)$, où $G$ est un $k$-groupe linéaire connexe lisse avec $\operatorname{Pic} \bar{G}=0$ (supposé réductif si $k$ est non parfait), et $H$ est un $k$-sous-groupe lisse de $G$ (non nécessairement connexe, mais de type (ssumult) ou avec $H^{0}$ réductif pour $k$ non parfait).

Une paire $(G, H)$ définit un espace homogène $X:=G / H$ avec un point marqué $x^{0}=e H \in X(k)$, où $e \in G(k)$ est l'élément neutre de $G$.

Par un morphisme de paires $\phi:\left(G_{1}, H_{1}\right) \rightarrow\left(G_{2}, H_{2}\right)$ on entend un homomorphisme surjectif $\phi: G_{1} \rightarrow G_{2}$ tel que $\phi\left(H_{1}\right)=H_{2}$. Si on pose $X_{1}=G_{1} / H_{1}$ et $X_{2}=G_{2} / H_{2}$, alors on a un morphisme $\phi_{*}:\left(X_{1}, x_{1}^{0}\right) \rightarrow\left(X_{2}, x_{2}^{0}\right)$, où $x_{1}^{0}$ et $x_{2}^{0}$ sont les points marqués correspondants.

4 e SÉRIE - TOME $46-2013-$ No $^{\circ}$ 
Soit $(G, H)$ une paire de $k$-groupes (avec $\operatorname{Pic} \bar{G}=0$ ). On choisit un plongement $i: S:=H^{\text {mult }} \rightarrow Q$ dans un tore quasi-trivial $Q$. On considère le plongement

$$
j: H \rightarrow G \times{ }_{k} Q, \quad h \mapsto(h, i(\mu(h))),
$$

où $\mu: H \rightarrow S$ est l'épimorphisme canonique. On pose $G_{Y}=G \times_{k} Q, H_{Y}=i_{*}(H)$. La paire $\left(G_{Y}, H_{Y}\right)$ définit un espace homogène $Y=G_{Y} / H_{Y}=\left(G \times_{k} Q\right) / i_{*}(H)$ avec un point marqué $y^{0}$. L'application de projection $\pi: G_{Y}=G \times Q \rightarrow G$ est surjective et satisfait $\pi\left(H_{Y}\right)=H$, et elle définit donc un morphisme de paires $\pi:\left(G_{Y}, H_{Y}\right) \rightarrow(G, H)$, qui à son tour définit un morphisme de variétés avec points marqués $\pi_{*}:\left(Y, y^{0}\right) \rightarrow\left(X, x^{0}\right)$. Notons encore $T_{Y}=G_{Y}^{\text {tor }}$ et $S_{Y}=H_{Y}^{\text {mult }}$. On remarque que l'application $j_{*}: S_{Y} \rightarrow T_{Y}$ est injective et que $\left(Y, \pi_{*}\right)$ est un torseur sur $X$ sous $Q$.

On construit une nouvelle paire $\left(G_{Z}, H_{Z}\right)$ comme suit: $G_{Z}=T_{Y} / j_{*}\left(S_{Y}\right), H_{Z}=1$, $Z=G_{Z}$. Alors $Z$ est un $k$-tore, on désigne par $z^{0}$ son élément neutre. On a un morphisme de paires $\nu:\left(G_{Y}, H_{Y}\right) \rightarrow\left(G_{Z},\{1\}\right)$ et le morphisme induit d'espaces homogènes $\nu_{*}:\left(Y, y^{0}\right) \rightarrow\left(Z, z^{0}\right)$. On a des quasi-isomorphismes

$$
\left.\left[\widehat{G_{Y}} \rightarrow \widehat{H_{Y}}\right] \rightarrow \widehat{T_{Y}} \rightarrow \widehat{S_{Y}}\right] \rightarrow\left[\widehat{G_{Z}} \rightarrow 0\right]
$$

On obtient des diagrammes
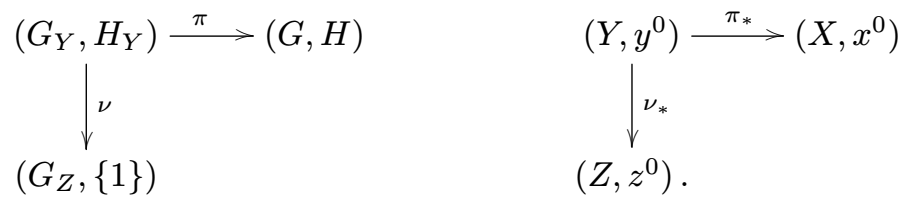

Lemme 6.4. - Le théorème 6.2 est valable pour l'espace principal homogène $Z$ sous $G_{Z}$.

Démonstration. - Soit $Z$ un $k$-tore. On a l'isomorphisme

$$
\Phi: \mathrm{Br}_{1, z^{0}} Z \rightarrow H^{2}(k, Z)
$$

de [9] et [10]. On a aussi l'isomorphisme

$$
\Phi^{S a}: \mathrm{Br}_{1, z^{0}} Z \rightarrow H^{2}(k, Z)
$$

de Sansuc [44], Lemme 6.9. Ces isomorphismes diffèrent par le signe, cf. [10], remarque 7.3 (sans preuve) et l'appendice A, proposition A.1 du présent article.

On a un diagramme commutatif

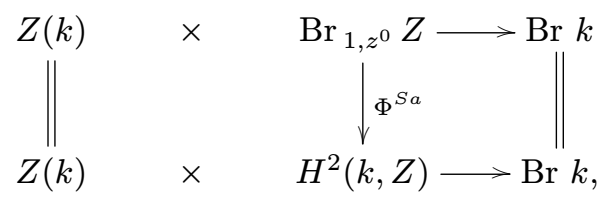

voir [44], (8.11.2). Ainsi pour $z \in Z(k), \alpha \in \mathrm{Br} Z$ on a

$$
\alpha(z)=z \cup \Phi^{S a}(\alpha)=-z \cup \Phi(\alpha),
$$

ce qui prouve le théorème 6.2 pour $Z$. 
Lemme 6.5. - Les diagrammes suivants sont commutatifs, toutes les flèches verticales marquées $(\cong)$ sont des isomorphismes, et l'application $\pi_{*}: Y(k) \rightarrow X(k)$ est surjective :

(8)
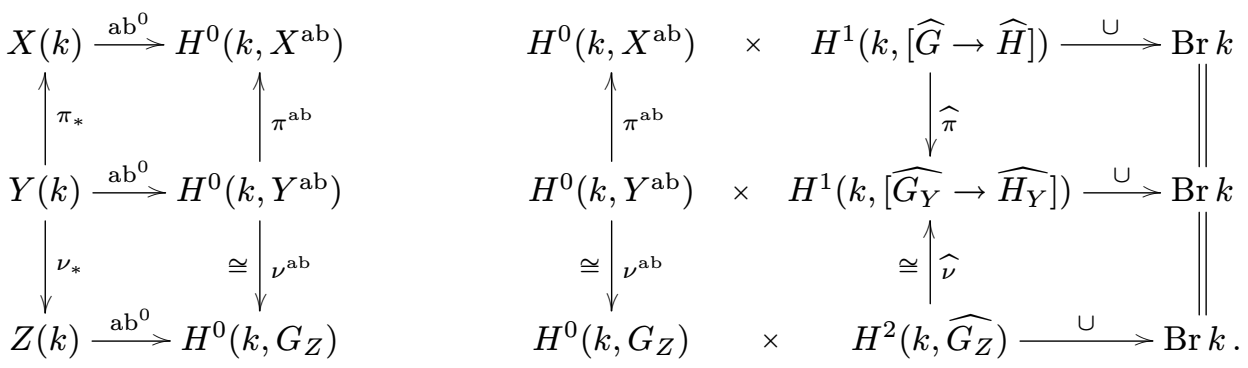

Démonstration. - Le lemme résulte de la fonctorialité de $a b^{0}$ et du cup produit et du fait que le morphisme de complexes de $k$-groupes de type multiplicatif

$$
\nu^{\mathrm{ab}}:\left[S_{Y} \rightarrow T_{Y}\right] \rightarrow\left[1 \rightarrow G_{Z}\right]
$$

est un quasi-isomorphisme. L'application $\pi_{*}: Y(k) \rightarrow X(k)$ est surjective parce que $Y$ est un torseur sur $X$ sous le tore quasi-trivial $Q$.

\section{Lemme 6.6. - Notons}

$$
\langle,\rangle: X(k) \times \mathrm{Br}_{1, x^{0}} X \rightarrow \mathrm{Br} k
$$

l'accouplement d'évaluation (et de même pour l'accouplement entre $Y(k)$ et $\operatorname{Br}_{1, y_{0}} Y$, ainsi que pour celui entre $Z(k)$ et $\left.\mathrm{Br}_{1, z^{0}} Z\right)$. Les diagrammes suivants sont commutatifs et toutes les flèches verticales marquées $(\cong)$ sont des isomorphismes :

(9)

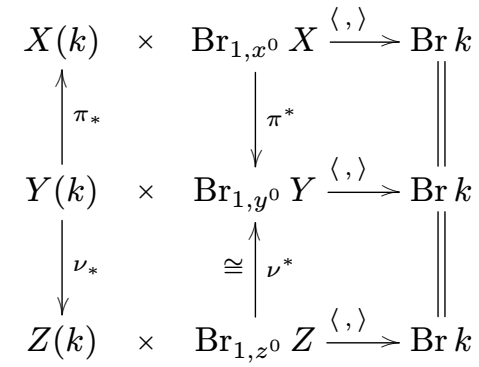

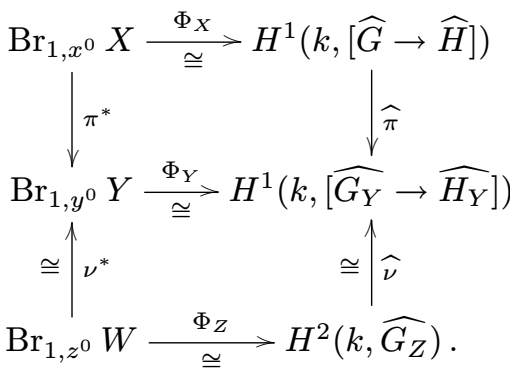

Démonstration. - La commutativité des diagrammes résulte de la fonctorialité de $\langle$,$\rangle et$ de $\Phi$. Comme $\widehat{\nu}$ est un isomorphisme, on voit que $\nu^{*}$ est un isomorphisme.

Preuve du Théorème 6.2. - Par «chasse au diagramme» dans les diagrammes (8) et (9) on réduit le théorème au lemme 6.4. 


\section{Corps globaux et corps finis}

Dans cette section, deux cas particuliers de corps de caractéristique $p>0$ sont traités : les corps globaux (théorème 7.4) et les corps finis (théorème 7.5). On notera que le théorème 7.4 fonctionne aussi pour un corps de nombres, mais fera l'objet d'une généralisation (utilisant le cas des corps finis) aux corps de caractéristique zéro arbitraires dans la section suivante (théorème 8.1).

Il semble raisonnable de conjecturer que cette généralisation vaut encore pour un corps quelconque de caractéristique $p>0$ si on se limite à la partie $p$-primaire des groupes considérés, mais on se heurte à une difficulté technique (voir la remarque après la preuve du théorème 8.1), ce qui fait que, dans cette section 7, nous avons dû nous limiter à des corps «arithmétiques».

Rappelons tout d'abord un théorème démontré par Douai dans sa thèse [26] :

Theorème 7.1 (Douai). - Soit K un corps local. Soit G un K-groupe semi-simple et soit $L$ un lien localement représentable pour la topologie étale (resp. fppf) par G. Alors tous les éléments de $H^{2}(K, L)$ (resp. de $H_{\mathrm{fppf}}^{2}(K, L)$ ) sont neutres.

Pour la preuve de ce résultat, on montre d'abord que le lien $L$ est globalement représentable par une $K$-forme de $G$ ([25], Lemme 1.1), ce qui permet de se ramener au cas où $L$ est le lien canoniquement associé à $G$. On utilise alors le fait que pour un groupe semisimple simplement connexe $R$ de centre $Z$, l'application bord $H^{1}(K, R / Z) \rightarrow H^{2}(K, Z)$ est surjective ([25], Th. 1.1, ou [3] Lemme 5.7 en caractéristique zéro), ce qui permet de conclure via [28], Cor. 3.8.

On en déduit une généralisation d'un résultat du deuxième auteur (cf. [24], Prop. 2.18 et [20], Prop. 4.2.20 joints au fait que si $K$ est un corps local, alors $H_{\text {fppf }}^{3}(K, \mu)=0$ pour tout $K$-groupe fini $\mu$, voir [41], Prop. 6.4) :

Proposition 7.2. - Soit $K$ un corps local (de caractéristique quelconque). Soit $G$ un $K$-groupe réductif vérifiant $\operatorname{Pic} \bar{G}=0$ et soit $H$ un $K$-sous-groupe de $G$ vérifiant : il existe des suites exactes

$$
\begin{gathered}
1 \rightarrow H^{\prime} \rightarrow H \rightarrow S \rightarrow 1 \\
1 \rightarrow U \rightarrow H^{\prime} \rightarrow H^{\mathrm{ss}} \rightarrow 1
\end{gathered}
$$

avec : $S$ de type multiplicatif, $H^{\mathrm{ss}}$ semi-simple, et $U$ sous-groupe unipotent distingué de $H$. Soit $T=G^{\text {tor }}$ le quotient torique maximal de $G$. Alors l'application canonique

$$
\mathrm{ab}_{K}: H_{\mathrm{fppf}}^{0}(K,[H \rightarrow G]) \rightarrow H_{\mathrm{fppf}}^{0}(K,[S \rightarrow T])
$$

est surjective. 
Remarque. - Si $K$ est de caractéristique zéro, l'hypothèse sur $H$ signifie exactement que $H$ est de type (ssumult). Noter aussi qu'on ne demande pas ici que $S$ soit lisse.

Démonstration. - On utilise [24], lemme 2.1 et remarque 2.2, pour obtenir un diagramme commutatif :

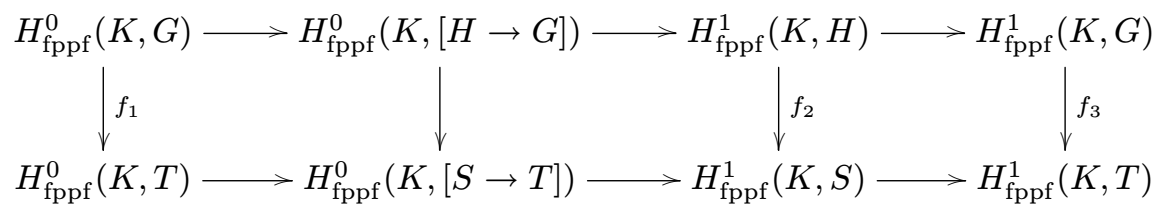

où la première ligne est une suite exacte d'ensembles pointés et la deuxième une suite exacte de groupes abéliens. Par ailleurs on a une action du groupe $H_{\mathrm{fppf}}^{0}(K, G)$ sur l'ensemble pointé $H_{\mathrm{fppf}}^{0}(K,[H \rightarrow G])$, qui est compatible via le diagramme ci-dessus avec l'action du groupe abélien $H_{\text {fppf }}^{0}(K, T)$ sur le groupe abélien $H_{\text {fppf }}^{0}(K,[S \rightarrow T])$ par translation (loc. cit., Prop. 2.6). Ceci permet par «chasse au diagramme » de se ramener à prouver les propriétés suivantes: $f_{1}$ et $f_{2}$ sont surjectives, $f_{3}$ a un noyau trivial. Comme $\operatorname{Pic} \bar{G}=0$, le sous-groupe dérivé $G^{\text {ss }}$ de $G$ est semi-simple simplement connexe, ce qui implique $H^{1}\left(K, G^{\text {ss }}\right)=1$. Ceci donne la surjectivité de $f_{1}$ et la trivialité du noyau de $f_{3}$.

Il reste à prouver que $f_{2}$ est surjective. Soit $H_{1}=H / U$, on a alors $S=H_{1} / H^{\text {ss }}$ et il suffit de vérifier que les flèches $u_{1}: H_{\mathrm{fppf}}^{1}(K, H) \rightarrow H_{\mathrm{fppf}}^{1}\left(K, H_{1}\right)$ et $u_{2}: H_{\mathrm{fppf}}^{1}\left(K, H_{1}\right) \rightarrow$ $H_{\mathrm{fppf}}^{1}(K, S)$ sont surjectives. Montrons que $u_{2}$ est surjective. Soit $\xi \in H_{\mathrm{fppf}}^{1}(K, S)$, alors l'obstruction à relever $\xi$ dans $H_{\mathrm{fppf}}^{1}\left(K, H_{1}\right)$ est un élément $\mathrm{ob}(\xi) \in H_{\mathrm{fppf}}^{2}(K, L)$, où $L$ est un lien localement représenté par $H^{\text {ss }}$ (voir [3], Cor. 6.4 en caractéristique zéro et la proposition IV.4.2.8(i) de [27] dans le cas général). Par le théorème 7.1 appliqué à $H^{\text {ss }}$, l'élément $\mathrm{ob}(\xi) \in H_{\mathrm{fppf}}^{2}(K, L)$ est neutre, donc $\xi$ provient de $H_{\mathrm{fppf}}^{1}\left(K, H_{1}\right)$ et la flèche $u_{2}$ est bien surjective.

La surjectivité de $u_{1}$ est quant à elle démontrée par Oesterlé : voir [42], IV.2.2, dans le cas où $U$ et $H$ sont lisses. On vérifie que la même preuve fonctionne dans le cas plus général où on ne suppose plus que les groupes soient lisses, en utilisant le fait que $H_{\mathrm{fppf}}^{2}\left(K, \alpha_{p}\right)=0$. Cela conclut la preuve.

Proposition 7.3. - Soit $K$ un corps local. Soit $G$ un $K$-groupe réductif vérifiant $\operatorname{Pic} \bar{G}=0$ et soit $H$ un $K$-sous-groupe lisse de $G$ vérifiant les hypothèses de la proposition 7.2. On pose $X=G / H$. Soit $\alpha$ un élément de $\operatorname{Br}_{1} X$ qui vérifie $\alpha(P)=0$ pour tout $P \in X(K)$. Alors $\alpha=0$.

Démonstration. - On note déjà que $\alpha \in \mathrm{Br}_{1, e} X$ et que $H$ est de type (ssumult). Soient $T=G^{\text {tor }}$ le quotient torique maximal de $G$ et $S=H^{\text {mult }}=H / H^{\prime}$ le quotient maximal de $H$ qui est de type multiplicatif.

D'après le théorème 6.2(a), on a un isomorphisme $\Phi_{X}: \operatorname{Br}_{1, e} X \rightarrow H^{1}(K,[\widehat{T} \rightarrow \widehat{S}])$. On sait que l'application d'évaluation

$$
\tau_{K}: X(K) \rightarrow H_{\text {fppf }}^{0}(K,[H \rightarrow G]) ; P \mapsto[[X]](P)
$$

est bijective. Il en résulte que l'image de l'application d'évaluation

$$
\mathrm{ab}_{K}^{0}: X(K) \rightarrow H_{\mathrm{fppf}}^{0}(K,[S \rightarrow T])
$$

$4{ }^{\text {e }}$ SÉRIE - TOME $46-2013$ - No 4 
est aussi l'image de

$$
\operatorname{ab}_{K}: H_{\mathrm{fppf}}^{0}(K,[H \rightarrow G]) \rightarrow H_{\mathrm{fppf}}^{0}(K,[S \rightarrow T]) .
$$

La proposition 7.2 dit que $a b_{K}$, et donc aussi $a b_{K}^{0}$, est surjective.

Posons alors $a=\Phi_{X}(\alpha) \in H^{1}(K,[\widehat{T} \rightarrow \widehat{S}])$. D'après le théorème 6.2(b), on obtient que $a$ vérifie

$$
a \cup \mathrm{ab}_{K}^{0}(P)=-\alpha(P)=0
$$

pour tout $P \in X(K)$. Comme $\mathrm{ab}_{K}^{0}$ est surjective, on obtient que $a$ est orthogonal à $H_{\mathrm{fppf}}^{0}(K,[S \rightarrow T])$ pour l'accouplement local (1). La proposition 3.2 donne alors $a=0$ d'où $\alpha=0$.

On en déduit une formule pour le groupe de Brauer non ramifié algébrique sur un corps global.

Theorème 7.4. - Soit $k$ un corps global de caractéristique $p \geq 0$. Soit $G$ un k-groupe réductif vérifiant $\operatorname{Pic} \bar{G}=0$ et soit $H$ un $k$-sous-groupe lisse de $G$ vérifiant les hypothèses de la proposition 7.2. On pose $X=G / H$. Soient $T=G^{\text {tor }}$ le quotient torique maximal de $G$ et $S=H^{\text {mult }}=H / H^{\prime}$ le quotient maximal de $H$ qui est de type multiplicatif.

(a) L'application $r d u \S 4$ induit pour $l \neq p$ un morphisme injectif entre les groupes de torsion $\ell$-primaires

$$
\left(\mathrm{Br}_{\mathrm{nr} 1} X / \mathrm{Br} k\right)\{\ell\} \rightarrow \amalg_{\omega}^{1}(k,[\widehat{T} \rightarrow \widehat{S}])\{\ell\}
$$

où on a noté $\mathrm{Br}_{\mathrm{nr}}{ }_{1} X:=\mathrm{Br}_{\mathrm{nr}} X \cap \mathrm{Br}_{1} X$.

(b) Si on suppose de plus que $X$ possède une compactification lisse $X^{c}$, l'application $r$ induit un isomorphisme

$$
\mathrm{Br}_{1} X^{c} / \mathrm{Br} k \rightarrow \amalg_{\omega}^{1}(k,[\widehat{T} \rightarrow \widehat{S}]) .
$$

(c) Sous les hypothèses de b), si l'on suppose de plus que le groupe H est connexe (réductif si $p>0$ ), l'application $r$ induit un isomorphisme

$$
\left(\operatorname{Br} X^{c} / \operatorname{Br} k\right)\left\{p^{\prime}\right\} \rightarrow \amalg_{\omega}^{1}(k,[\widehat{T} \rightarrow \widehat{S}])\left\{p^{\prime}\right\} .
$$

Preuve du théorème 7.4. - Plaçons-nous sous les hypothèses de b). On identifie le groupe $\mathrm{Br}_{1} X^{c} / \mathrm{Br} k$ à $\mathrm{Br}_{1, e} X^{c}$. D'après le théorème 6.2(a), on a un isomorphisme $\Phi_{X}: \mathrm{Br}_{1, e} X \rightarrow$ $H^{1}(k,[\widehat{T} \rightarrow \widehat{S}])$, et la proposition 4.1 donne déjà que l'image de $\mathrm{Br}_{1, e} X^{c}$ par $\Phi_{X}$ contient $\amalg_{\omega}^{1}(k,[\widehat{T} \rightarrow \widehat{S}])$. Il reste à montrer l'inclusion inverse.

Fixons $\alpha \in \mathrm{Br}_{1, e} X^{c} \subset \mathrm{Br}_{1, e} X$. Comme on l'a déjà vu (proposition 4.2), il existe un ensemble fini de places $\Sigma$ de $k$ (contenant les éventuelles places archimédiennes) tel que $\alpha\left(P_{v}\right)=0$ pour toute $v \notin \Sigma$ et tout point local $P_{v} \in X\left(k_{v}\right)$. On conclut alors en appliquant la proposition 7.3 à tous les localisés $\alpha_{v} \in \operatorname{Br}_{1, e}\left(X \times_{k} k_{v}\right)$ pour $v \notin \Sigma$.

$\mathrm{Si}$ on se place sous les hypothèses de $\mathrm{c})$, le théorème 5.1 donne de plus $\left(\operatorname{Br} X^{c}\right)\left\{p^{\prime}\right\}=$ $\left(\mathrm{Br}_{1} X^{c}\right)\left\{p^{\prime}\right\}$, d'où la conclusion.

Enfin, la preuve de a) est identique à celle de b) en utilisant la proposition 4.2 dans le cas où on ne suppose pas l'existence d'une compactification lisse. 
Remarque. - Il ne semble pas clair que pour un complexe quelconque $[S \rightarrow T]$ de groupes de type multiplicatif sur un corps de nombres, les groupes $\amalg_{\omega, \text { alg }}^{1}\left(C^{\bullet}\right)$ et $\amalg_{\omega}^{1}\left(C^{\bullet}\right)$ coïncident toujours; ce sera vrai a posteriori (via le théorème 8.1 ci-dessous) pour un complexe provenant d'un espace homogène comme dans le théorème 7.4. On peut aussi vérifier cette égalité assez facilement si $S$ et $T$ sont des tores, ou bien si $T$ est un tore quasi-trivial.

Theorème 7.5. - Soit $k$ un corps fini de caractéristique p. Soit G un k-groupe réductif. Soit $X$ un espace homogène de $G$, de stabilisateur géométrique $\bar{H}$ lisse. On suppose que l'une des deux hypothèses suivantes est satisfaite :

(i) le $\bar{k}$-groupe $\bar{H}$ est connexe.

(ii) $\bar{H}$ est de type (ssumult) et $\operatorname{Pic} \bar{G}=0$.

Alors $\mathrm{Br}_{\mathrm{nr}}{ }_{1} X\{\ell\}=0$ pour tout nombre premier $\ell \neq$ Car $k$.

Si on suppose de plus qu'on a une compactification lisse $X^{c}$ de $X$, on a $\mathrm{Br}_{1} X^{c}=0$.

Dans le cas ( $i)$, si $\bar{H}$ est réductif, on a en outre $\left(\operatorname{Br} X^{c}\right)\left\{p^{\prime}\right\}=0$.

Remarque. - Dans le point (ii), l'hypothèse Pic $\bar{G}=0$ est nécessaire, comme on peut le voir dans l'exemple de la proposition 8.5 , où le groupe $\bar{H}$ est fini de type multiplicatif et $\operatorname{Br}_{\text {nr } 1}(X)\left\{p^{\prime}\right\} \neq 0$.

Démonstration. - Notons déjà que $\mathrm{Br} k=0$ ([47], section II.3., corollaire à la proposition 8). D'autre part comme $G$ est linéaire connexe et $k$ fini (donc $C_{1}$ ), l'espace homogène $X$ possède un point rationnel par les théorèmes de Steinberg et de Springer ([47], section III.2., corollaire au théorème 3 , ou [49], 3.10; dans le cas particulier d'un corps fini ce résultat est dû à Lang). Ainsi on peut supposer $X=G / H$, où $H$ est un $k$-sous-groupe de $G$ de type (ssumult) (on vérifie facilement que si $H \times_{k} \bar{k}$ est de type (ssumult), alors $H$ est de type (ssumult)), qui est de plus supposé connexe si l'on ne fait pas l'hypothèse Pic $\bar{G}=0$.

Soient alors $K=k((t))$ et $\vartheta_{K}=k[[t]]$. On commence par le cas où Pic $\bar{G}=0$. Posons $T=G^{\text {tor }}$. Comme $H$ est de type (ssumult), on a une suite exacte de $k$-groupes lisses

$$
1 \rightarrow H^{\prime} \rightarrow H \rightarrow S \rightarrow 1
$$

avec $S$ de type multiplicatif lisse, et $H^{\prime}$ connexe lisse sans caractères. Comme $k$ est parfait, le groupe $H^{\prime}$ est alors extension d'un $k$-groupe semi-simple par un $k$-groupe unipotent $U$ connexe et lisse. Par ailleurs $U$ reste distingué dans $H$ car $\bar{U}$ est invariant par tout $\bar{k}$-automorphisme de $\bar{H}^{\prime}$ (ce qui suffit vu que tous les groupes considérés sont lisses) en tant que radical unipotent de $\bar{H}^{\prime}$. Il en résulte que le groupe $H_{K}:=H \times_{k} K$ satisfait aux hypothèses de la proposition 7.3. Par ailleurs l'application $\operatorname{Br} X \rightarrow \operatorname{Br} X_{K}$ est injective car la restriction $\mathrm{Br} X_{\vartheta_{K}} \rightarrow \operatorname{Br} X_{K}$ est injective ( $X_{\vartheta_{K}}$ et $X_{K}$ sont deux schémas intègres réguliers qui ont même corps de fonctions), ainsi que $\operatorname{Br} X \rightarrow \operatorname{Br} X_{\emptyset_{K}}$ qui possède une rétraction via le morphisme d'évaluation en zéro $\theta_{K} \rightarrow k$. Il suffit donc de montrer que tout élément $\alpha \in \mathrm{Br}_{\mathrm{nr}}{ }_{1} X\{\ell\}$ pour $l \neq p$ (resp. tout $\alpha \in \mathrm{Br}_{1} X^{c}$ si on suppose l'existence d'une compactification lisse $X^{c}$ ) a une restriction $\alpha_{K}$ nulle dans $\operatorname{Br}_{1}\left(X_{K}\right)$. Pour cela, on observe que pour tout $K$-point $P$ de $X$, on a $\alpha_{K}(P)=0$ via la proposition 4.3, et on conclut alors avec la proposition 7.3 ; nous remercions $\mathrm{O}$. Wittenberg qui nous a signalé cette possibilité d'utiliser directement le corps $k((t))$; notre argument initial était plus compliqué et passait par le corps global $k(t)$. 
Si on ne suppose plus $\operatorname{Pic} \bar{G}=0$, on observe ([16], lemme 1.5) que $X$ est isomorphe à un espace homogène $G_{1} / H_{1}$, où $G_{1}$ est réductif connexe quasi-trivial (en particulier $\operatorname{Pic} \bar{G}_{1}=0$ ) et $H_{1}$ est extension centrale de $H$ par un $k$-tore $T_{1}$; en particulier $H_{1}$ reste lisse et connexe et on est ramené au cas précédent.

Le dernier point de l'énoncé, sous l'hypothèse $\bar{H}$ réductif, est alors une conséquence du théorème 5.1 .

Remarque. - L'égalité $\mathrm{Br}_{\mathrm{nr} 1} X\left\{p^{\prime}\right\}=0$ est valable sans supposer $G$ réductif, en utilisant le même argument que dans la preuve du théorème 8.1 ci-dessous (comme il s'agit d'un argument birationnel, il ne marche pas pour la $p$-partie).

\section{Corps de caractéristique zéro}

Dans cette section, nous allons suivre la méthode de Colliot-Thélène et Kunyavskiī [15] pour établir, par réduction au cas des corps finis, le

Theorème 8.1. - Soit $k$ un corps de caractéristique zéro. Soit $G$ un k-groupe linéaire connexe vérifiant $\operatorname{Pic} \bar{G}=0$, on note $T=G^{\text {tor }}$ son quotient torique maximal. Soit $X$ un espace homogène de $G$ de stabilisateur géométrique $\bar{H}$, on suppose que $\bar{H}$ est de type (ssumult) et on note $S$ le $k$-groupe de type multiplicatif canoniquement associé à $\bar{H}$. Soit $X^{c}$ une compactification lisse de $X$. Alors on a une suite exacte

$$
0 \rightarrow \operatorname{Br}_{1} X^{c} / \mathrm{Br} k \rightarrow \amalg_{\omega, \text { alg }}^{1}([\widehat{T} \rightarrow \widehat{S}]) \rightarrow \operatorname{ker}\left[H^{3}\left(k, \mathbf{G}_{m}\right) \rightarrow H^{3}\left(X, \mathbf{G}_{m}\right)\right]
$$

qui induit un isomorphisme

$$
\mathrm{Br}_{1} X^{c} / \mathrm{Br} k \simeq \amalg_{\omega, \text { alg }}^{1}([\widehat{T} \rightarrow \widehat{S}])
$$

si on suppose de plus $X(k) \neq \varnothing$ ou $H^{3}\left(k, \mathbf{G}_{m}\right)=0$.

Rappelons que bien que $\bar{H}$ ne soit pas forcément défini sur $k$, il est muni d'un $k$-lien qui induit une action de $\Gamma_{k}$ sur le quotient maximal de type multiplicatif $\bar{S}=\bar{H}^{\text {mult }}$ de $\bar{H}$, et donc une $k$-forme canonique $S$ de $\bar{S}$.

Démonstration. - D'après le théorème 6.2 (a) (théorème 1 de [10]), on sait que $K D^{\prime}(X)$ est isomorphe canoniquement à $[\widehat{T} \rightarrow \widehat{S}]$ dans la catégorie dérivée, donc il suffit de construire une suite exacte

$$
0 \rightarrow \mathrm{Br}_{1} X^{c} / \mathrm{Br} k \rightarrow \amalg_{\omega, \text { alg }}^{1}\left(K D^{\prime}(X)\right) \rightarrow \operatorname{ker}\left[H^{3}\left(k, \mathbf{G}_{m}\right) \rightarrow H^{3}\left(X, \mathbf{G}_{m}\right)\right] .
$$

On sait déjà par la proposition 4.1 qu'on a une suite exacte (dont le dernier terme est zéro si $X(k) \neq \varnothing)$

$$
0 \rightarrow \mathrm{Br}_{1} X / \mathrm{Br} k \rightarrow H^{1}\left(k, K D^{\prime}(X)\right) \rightarrow \operatorname{ker}\left[H^{3}\left(k, \mathbf{G}_{m}\right) \rightarrow H^{3}\left(X, \mathbf{G}_{m}\right)\right]
$$

telle que de plus l'image de $\operatorname{Br}_{1} X^{c} / \operatorname{Br} k$ dans $H^{1}\left(k, K D^{\prime}(X)\right)$ contienne le noyau

$$
\operatorname{ker}\left[\amalg_{\omega, \operatorname{alg}}^{1}\left(k, K D^{\prime}(X)\right) \rightarrow H^{3}\left(k, \mathbf{G}_{m}\right)\right],
$$

voir le diagramme (2) dans la preuve de la proposition 4.1. Comme cette image est contenue dans celle de $H^{1}\left(k, K D^{\prime}\left(X^{c}\right)\right)=H^{1}\left(k, \operatorname{Pic} \overline{X^{c}}\right)$, il ne reste plus qu'à montrer que

$$
H^{1}\left(k, \operatorname{Pic} \overline{X^{c}}\right)=\amalg_{\omega, \text { alg }}^{1}\left(k, \operatorname{Pic} \overline{X^{c}}\right) .
$$


Traitons d'abord le cas où $G$ est réductif et où $X$ possède un $k$-point en démontrant la

Proposition 8.2. - Avec les notations ci-dessus, supposons de plus que le groupe G est réductif et que $X(k) \neq \varnothing$. Alors pour tout sous-groupe procyclique $\Gamma_{1}$ de $\Gamma_{k}$, on a

$$
H^{1}\left(\Gamma_{1}, \operatorname{Pic} \overline{X^{c}}\right)=0 .
$$

Preuve de la proposition 8.2. - L'argument va consister à se ramener au cas où $k$ est fini; c'est essentiellement le même que pour la proposition 2 de [15], nous le reproduisons en entier pour le confort du lecteur.

On sait que $\bar{G}:=G \times_{k} \bar{k}$ est une $\bar{k}$-variété rationnelle ([2], corollaires 14.14 et 18.8). De ce fait, $\bar{X} \simeq \bar{G} / \bar{H}$ (et donc aussi $\bar{X}^{c}$ ) est une variété $\bar{k}$-unirationnelle puisqu'on a un $\bar{k}$-morphisme dominant $\bar{G} \rightarrow \bar{X}$. Comme la caractéristique de $k$ est nulle, ceci implique que pour tout $i>0$, les groupes $H^{i}\left(\bar{X}^{c}, \vartheta_{\bar{X}^{c}}\right.$ ) (ou encore les groupes $H^{i}\left(X^{c}, \vartheta_{X^{c}}\right)$ ) sont nuls. Rappelons qu'on obtient ce résultat (bien connu) en se ramenant au cas $k=\mathbf{C}$ par le principe de Lefschetz, puis en utilisant le fait que $H^{i}\left(X^{c}, \theta_{X^{c}}\right)=H^{0}\left(X^{c}, \Omega_{X^{c}}^{i}\right)$ via la théorie de Hodge (c'est cet argument qui ne marche pas en caractéristique $p$ ). La nullité de ces groupes est alors assurée par le fait que $\bar{X}^{c}$ est dominé par un espace projectif, car on a $H^{i}\left(\mathbf{P}^{r}, \theta_{\mathbf{P}^{r}}\right)=0$ pour tous $i, r>0$ via [35], théorème III.2.7 et théorème III.5.1(b),(d). La propriété que $\bar{X}^{c}$ est unirationnelle implique également que $\mathrm{Pic} \bar{X}^{c}$ est sans torsion (par exemple parce que d'après un théorème de Serre [45], le groupe fondamental géométrique $\pi_{1}\left(\bar{X}^{c}\right)$ est nul, ce qui donne que le groupe $\left(\operatorname{Pic} \bar{X}^{c}\right)[n] \simeq H^{1}\left(\bar{X}^{c}, \mathbf{Z} / n\right)$ est nul); le fait que $H^{1}\left(X^{c}, \theta_{X^{c}}\right)=0$ implique que $\operatorname{Pic} \bar{X}^{c}$ est de type fini (car égal au groupe de Néron-Severi de $\left.\bar{X}^{c}\right)$.

Soit maintenant $\tilde{k} / k$ une extension finie galoisienne déployant $\operatorname{Pic} \bar{X}^{c}$, posons $\Gamma=\operatorname{Gal}(\tilde{k} / k)$. Le fait que le groupe abélien $\operatorname{Pic} \bar{X}^{c}$ soit libre et de type fini implique $H^{1}\left(\tilde{k}, \mathrm{Pic} \bar{X}^{c}\right)=0$. Il suffit alors pour montrer la proposition de vérifier que pour tout sousgroupe cyclique $\gamma \subset \Gamma$, on a $H^{1}\left(\gamma, \operatorname{Pic} X_{\tilde{k}}^{c}\right)=0$ (où $X_{\tilde{k}}^{c}=X^{c} \times_{k} \tilde{k}$ ). On écrit $\tilde{k}=k[t] / P(t)$, où $P \in k[t]$ est un polynôme irréductible. Comme $X \hookrightarrow X^{c}$ est un morphisme de $k$-schémas de type fini, on peut trouver un corps $k_{0} \subset k$ de type fini sur $\mathbf{Q}$ sur lequel $G, X, X^{c}$, et le plongement $X \rightarrow X^{c}$ sont définis; comme $X(k) \neq \varnothing$ par hypothèse, on peut également (quitte à remplacer $k_{0}$ par le corps résiduel de $x \in X_{k_{0}}$ défini comme l'image d'un $k_{0}$-morphisme Spec $k \rightarrow X_{k_{0}}$ ) supposer que $X\left(k_{0}\right) \neq \varnothing$ (ce qui implique que $X_{k_{0}}$ est isomorphe à un quotient $G_{k_{0}} / H_{k_{0}}$, où $H_{k_{0}}$ est un $k_{0}$-groupe de type (ssumult)). Quitte à agrandir $k_{0}$ dans $k$, on peut aussi supposer que $P(t) \in k_{0}[t]$ et que $\tilde{k}_{0}:=k_{0}[t] / P(t)$ déploie $\mathrm{Pic} \bar{X}^{c}$ (qui est de type fini). On peut enfin supposer que $\tilde{k}_{0}$ est galoisienne sur $k_{0}$ : en effet si $a \in \tilde{k}_{0}$ est une racine de $P$, on a $\tilde{k}_{0}=k_{0}[a]$ et $\tilde{k}=k[a]$; toute racine $a_{i}$ de $P$ s'écrit alors $Q_{i}(a)$ avec $Q_{i} \in k[t]$ (parce que $\tilde{k}$ est galoisienne sur $k$ ), et il suffit alors de prendre $k_{0}$ assez grand pour que tous les polynômes $Q_{i}$ soient dans $k_{0}[t]$. L'irréductibilité de $P$ sur $k_{0}$ implique alors que $\operatorname{Gal}(\tilde{k} / k)$ est canoniquement isomorphe à $\operatorname{Gal}\left(\tilde{k}_{0} / k_{0}\right)$, et par ailleurs les inclusions

$$
\operatorname{Pic} X_{\tilde{k}_{0}}^{c} \rightarrow \operatorname{Pic} X_{\tilde{k}}^{c} \rightarrow \operatorname{Pic} \bar{X}^{c}
$$

sont des isomorphismes. Ainsi le $\operatorname{Gal}(\tilde{k} / k)$-module $\operatorname{Pic} X_{\tilde{k}}^{c}$ est canoniquement isomorphe au $\operatorname{Gal}\left(\tilde{k}_{0} / k_{0}\right)$-module $\operatorname{Pic} X_{\tilde{k}_{0}}^{c}$. Pour simplifier les notations, on notera dans la suite $k=k_{0}$ et $\tilde{k}=\tilde{k}_{0}$ (en particulier on s'est ramené au cas où $X=G / H$ avec $H$ de type (ssumult)). 
On prend alors un modèle entier de toute la situation : comme $k$ est un corps de type fini sur $\mathbf{Q}$, on peut trouver un anneau intègre et régulier $A$ de type fini sur $\mathbf{Z}$, de corps des fractions $k$, tel que la fermeture intégrale $\widetilde{A}$ de $A$ dans $\tilde{k}$ soit finie, étale, galoisienne de groupe $\Gamma \operatorname{sur} A$, et des $A$-schémas de type fini $\mathscr{G}, \mathcal{H}, \chi=\mathscr{G} / \mathcal{H}, \chi^{c}$ de fibres génériques respectives $G, H, X, X^{c}$, tels que :

(i) $\mathscr{G}$ est un $A$-schéma en groupes réductif (en particulier lisse à fibres connexes).

(ii) $\mathcal{H}$ est un $A$-schéma en groupes lisse et $\chi$ est un $A$-schéma lisse.

(iii) $\chi^{c}$ est propre et lisse sur $A$ et on a une $A$-immersion ouverte $\chi \rightarrow \chi^{c}$ qui étend $X \rightarrow X^{c}$.

(iv) Pour tout point $x \operatorname{de} \operatorname{Spec} A$, de corps résiduel $\kappa(x)$, la fibre $\chi_{x} \subset \chi_{x}^{c}$ est une compactification lisse de $\chi_{x}$ sur $\kappa(x)$.

On utilise alors que $H^{1}\left(X^{c}, \theta_{X^{c}}\right)=H^{2}\left(X^{c}, \theta_{X^{c}}\right)=0$. Le théorème de semi-continuité ([35], III.12.8) nous permet de supposer (quitte à restreindre encore $\operatorname{Spec} A$ ) que pour tout point $x$ de Spec $A$, on a encore $H^{1}\left(\chi_{x}^{c}, \theta_{\chi_{x}^{c}}\right)=H^{2}\left(\chi_{x}^{c}, \theta_{\chi_{x}^{c}}\right)=0$. Les théorèmes de Grothendieck sur les faisceaux inversibles ([29], paragraphe 5; voir aussi [31], Prop. 3.4.2) disent alors qu'on a un isomorphisme de spécialisation $\operatorname{Pic} X^{c} \simeq \operatorname{Pic} \chi_{x}^{c}$; plus précisément le foncteur $\underline{\mathrm{Pic}} \chi^{c} / A$ est représenté par un $A$-groupe constant tordu localement libre pour la topologie étale, et on peut supposer (quitte à restreindre encore $A$ et à augmenter $\tilde{k}$ et $\widetilde{A}$ ) que ce $A$-groupe est déployé (autrement dit devient isomorphe à $\mathbf{Z}^{n}$ ) sur $\operatorname{Spec} \widetilde{A}$.

Quitte à restreindre encore $\operatorname{Spec} A$, on peut aussi supposer que les fibres de $\mathscr{G}$ ont un groupe de Picard géométrique nul (en effet sur un ouvert $U$ de $\operatorname{Spec} A$ on a un revêtement simplement connexe $\mathscr{G}^{\text {sc }}$ de $\mathscr{G}^{\text {ss }}$, qui est un revêtement fini étale de $\mathscr{G}^{\text {ss }}$ trivial au point générique, donc sur $U$ tout entier). De même on peut supposer que les fibres géométriques de $\mathcal{H}$ sont de type (ssumult) (car $H$ est extension d'un $k$-groupe de type multiplicatif par un groupe unipotent et ces propriétés s'étendent sur un certain ouvert $\operatorname{de} \operatorname{Spec} A$ ).

Soit maintenant $\gamma$ un sous-groupe cyclique de $\Gamma$ (correspondant à une sous-extension $k_{1} \supset k$ de $\left.\tilde{k}\right)$ et $A_{1}:=\widetilde{A}^{\gamma}$ le sous-anneau de $\widetilde{A}$ des invariants pour l'action de $\gamma$. Le morphisme $\rho: \operatorname{Spec} \widetilde{A} \rightarrow \operatorname{Spec} A_{1}$ est un revetement étale cyclique de groupe $\gamma$, et les anneaux $\widetilde{A}$ et $A_{1}$ sont intègres, réguliers, de type fini sur $\mathbf{Z}$. La version de Serre du théorème de Čebotarev ([46], théorème 7) dit alors qu'il existe un idéal maximal (en fait une infinité) $m$ de $A_{1}$ tel que la fibre en $m$ de $\rho$ soit du type Spec $\widetilde{F}$, où $\widetilde{F}$ est un corps fini (extension finie du corps $\left.F_{1}:=A_{1} / m\right)$, autrement dit il y a un seul point $\widetilde{m}$ de Spec $\widetilde{A}$ au-dessus de $m$ et on a $\gamma:=\operatorname{Gal}\left(\tilde{k} / k_{1}\right)=\operatorname{Gal}\left(\widetilde{F} / F_{1}\right)$. Soit $Y=\chi^{c} \times_{A} F_{1}$ la fibre de $\chi^{c} \times_{A} A_{1}$ en $m$. Comme $\underline{\mathrm{Pic}} \chi^{c} / A_{A} \times{ }_{A} \widetilde{A}$ est un schéma en groupes constant, sa fibre générique est isomorphe à sa fibre en $\widetilde{m}$ et on obtient

$$
H^{1}\left(\gamma, \operatorname{Pic} X_{\tilde{k}}^{c}\right)=H^{1}\left(\gamma, \operatorname{Pic}\left(Y \times_{F} \widetilde{F}\right)\right) .
$$

$\operatorname{Or} \operatorname{Gal}(\bar{F} / \widetilde{F})$ agit trivialement sur $\operatorname{Pic} \bar{Y}$, donc $H^{1}\left(\gamma, \operatorname{Pic}\left(Y \times_{F} \widetilde{F}\right)\right)$ est un sous-groupe de $H^{1}\left(F_{1}\right.$, Pic $\left.\bar{Y}\right)$. Comme $Y$ est une compactification lisse d'un espace homogène d'un $F_{1}$-groupe linéaire $\mathscr{G}_{m}$ connexe de groupe de Picard géométrique nul et à stabilisateur $\mathscr{H}_{m}$ de type (ssumult), le théorème 7.5 dit que $H^{1}\left(F_{1}, \operatorname{Pic} \bar{Y}\right)=\operatorname{Br}_{1} Y=0$, donc $H^{1}\left(\gamma, \operatorname{Pic} X_{\tilde{k}}^{c}\right)=0$, ce qui achève la preuve de la proposition. 
Fin de la preuve du théorème 8.1. - Supposons d'abord que $G$ est réductif (mais pas forcément que $X(k) \neq \varnothing)$. Pour se ramener au cas $X(k) \neq \varnothing$, on utilise l'" astuce du point générique » (comme dans [15], pages 46-47). Soit $K$ le corps des fonctions de $X$, alors $X(K) \neq \varnothing$. Soit $\bar{K}$ une clôture algébrique de $K$, alors $\bar{k}$ s'identifie avec la fermeture algébrique de $k$ dans $\bar{K}$, et on a $\bar{k} \cap K=k$ (les corps $K$ et $\bar{k}$ sont linéairement disjoints sur $k$ car $X$ est géométriquement intègre). Soit comme ci-dessus $\tilde{k}$ une extension galoisienne finie de $k$ dans $\bar{k}$ déployant $\operatorname{Pic} X_{\bar{k}}^{c}$, alors $\operatorname{Gal}(\tilde{k} / k)$ agit sur $\operatorname{Pic} X_{\bar{k}}^{c}$. On pose $\widetilde{K}=K \tilde{k} \subset \bar{K}$, alors $\widetilde{K}$ déploie $\operatorname{Pic} X_{\bar{K}}^{c}$, et $\operatorname{Gal}(\widetilde{K} / K)$ agit sur Pic $X_{\bar{K}}^{c}$. On a un isomorphisme canonique $\varepsilon: \operatorname{Gal}(\widetilde{K} / K) \stackrel{\sim}{\rightarrow} \operatorname{Gal}(\tilde{k} / k)$ et un isomorphisme canonique $\varepsilon$-équivariant $\operatorname{Pic} X_{\bar{k}}^{c} \stackrel{\sim}{\rightarrow} \operatorname{Pic} X_{\bar{K}}^{c}$. Comme $X(K) \neq \varnothing$, par la proposition 8.2, pour chaque sous-groupe cyclique $\Gamma_{1} \subset \operatorname{Gal}(\widetilde{K} / K)$ on a $H^{1}\left(\Gamma_{1}, \operatorname{Pic} X_{\bar{K}}^{c}\right)=$ 0 . On conclut que pour chaque sous-groupe cyclique $\gamma_{1} \subset \operatorname{Gal}(\tilde{k} / k)$ on a $H^{1}\left(\gamma_{1}, \operatorname{Pic} X_{\bar{k}}^{c}\right)=$ 0 . Ceci établit le théorème 8.1 pour $G$ réductif.

Passons enfin au cas général où on ne suppose plus $G$ réductif. Soient $G^{u}$ le radical unipotent de $G$ et $Y=X / G^{u}$ (voir [4], lemme 3.1 pour la représentabilité de ce quotient). Alors $Y$ est un espace homogène sous le $k$-groupe réductif $G^{\prime}:=G / G^{u}$, de stabilisateur géométrique $\bar{H}^{\prime}=\bar{H} /\left(\bar{H} \cap \bar{G}^{u}\right)$, pour lequel $\left(\bar{H}^{\prime}\right)^{\text {mult }}=\bar{H}^{\text {mult }}$ vu que $\left(\bar{H} \cap \bar{G}^{u}\right)$ est un groupe unipotent. On a encore $\operatorname{Pic} \bar{G}^{\prime}=0$ via [44] corollaire 6.11, et $\left(G^{\prime}\right)^{\text {tor }}=G^{\text {tor }}$ par définition de $G^{u}$. Il suffit donc, pour se ramener au cas $G$ réductif, de montrer que $\operatorname{Br}_{\mathrm{nr}}{ }_{1} Y=\mathrm{Br}_{\mathrm{nr}}{ }_{1} X$. Or ceci résulte de ce que $X$ est stablement $k$-birationnel à $Y$, par le même argument qu'à la fin de la deuxième étape dans la preuve du théorème 5.1.

Remarques. - - Quand $H$ est connexe, la même méthode donne encore $\operatorname{Br}_{1} X^{c} / \mathrm{Br} k \simeq$ $\amalg_{\omega, \text { alg }}^{1}\left(K D^{\prime}(X)\right)$ sans supposer que $\operatorname{Pic} \bar{G}=0$, mais $K D^{\prime}(X)$ n'a plus en général une description aussi agréable que quand $\operatorname{Pic} \bar{G}=0$, voir [23], th. 0.1 .

- Si on ne suppose plus $k$ de caractéristique zéro, mais si on suppose de plus $G$ réductif (et toujours $\bar{H}$ de type (ssumult)), la même méthode fonctionne à condition de savoir qu'on $a H^{1}\left(X^{c}, \vartheta_{X^{c}}\right)=H^{2}\left(X^{c}, \vartheta_{X^{c}}\right)=0$. Or ceci est une question ouverte en caractéristique $p$ pour les variétés séparablement unirationnelles comme $X^{c}$.

On déduit du théorème 8.1 le corollaire suivant, via le théorème 5.1 :

Corollaire 8.3. - Avec les notations et les hypothèses du théorème 8.1, si on suppose en outre que $\bar{H}$ est connexe, alors on a une suite exacte

$$
0 \rightarrow \operatorname{Br} X^{c} / \mathrm{Br} k \rightarrow \amalg_{\omega, \text { alg }}^{1}([\widehat{T} \rightarrow \widehat{S}]) \rightarrow \operatorname{ker}\left[H^{3}\left(k, \mathbf{G}_{m}\right) \rightarrow H^{3}\left(X, \mathbf{G}_{m}\right)\right]
$$

qui induit un isomorphisme

$$
\operatorname{Br} X^{c} / \mathrm{Br} k \simeq \amalg_{\omega, \text { alg }}^{1}([\widehat{T} \rightarrow \widehat{S}])
$$

si on suppose de plus $X(k) \neq \varnothing$ ou $H^{3}\left(k, \mathbf{G}_{m}\right)=0$.

Le théorème 8.1 n'est plus valable si on ne suppose pas $\bar{H}$ de type (ssumult). On trouve dans [21] un contre-exemple avec $G=\mathrm{SL}_{n}$ et $\bar{H}$ fini non commutatif. Nous allons pour conclure en déduire un contre-exemple similaire avec $\bar{H}$ extension d'un groupe fini commutatif par un tore, ce qui montre que l'hypothèse que le groupe des composantes connexes $\bar{H} / \bar{H}^{0}$ est commutatif n'est pas non plus suffisante. 
Soit $p$ un nombre premier. On considère le groupe fini $E$ considéré dans la proposition 6.1 de [21]. Il est donné par la présentation

$$
E=\left\langle x, y, z: x^{p^{2}}=y^{p^{2}}=z^{p^{2}}=1 ;[x, y]=z^{p}\right\rangle .
$$

C'est une extension centrale de $F=E^{\mathrm{ab}}=\mathbf{Z} / p^{2} \times \mathbf{Z} / p^{2} \times \mathbf{Z} / p$ par $A=D(E)=\mathbf{Z} / p$. Soit $k=\mathbf{Q}\left(\zeta_{p}\right)$, on regarde $E$ comme un groupe algébrique fini (constant) sur $k$. On plonge $\mathbf{Z} / p=\mu_{p}$ dans $\mathbf{G}_{m}$, et on définit un $k$-groupe algébrique $H$ par $H:=\left(E \times \mathbf{G}_{m}\right) / j(A)$, où $j: A \rightarrow E \times \mathbf{G}_{m}$ est le plongement diagonal (noter que $j(A)$ est bien distingué dans $E \times \mathbf{G}_{m}$ car $A$ est central dans $E$ ). On plonge ensuite $H$ dans un groupe $\mathrm{SL}_{n}$.

Proposition 8.4. - Soit $X$ l'espace homogène défini par $X=\mathrm{SL}_{n} / H$. Alors $\mathrm{Br}_{1, e} X=H^{1}\left(k, \widehat{H}^{\text {mult }}\right)$, mais $\mathrm{Br}_{\mathrm{nr} 1, e} X$ contient strictement $\amalg_{\omega}^{1}\left(\widehat{H}^{\text {mult }}\right)$.

Démonstration. - Le fait que $\mathrm{Br}_{1, e} X=H^{1}\left(k, \widehat{H}^{\text {mult }}\right)$ résulte du théorème 1 de [10] joint au fait que $\mathrm{SL}_{n}$ est sans caractères et toute fonction inversible sur $X$ est constante.

On a un diagramme commutatif, dont les deux premières lignes sont exactes et correspondent à des extensions centrales :

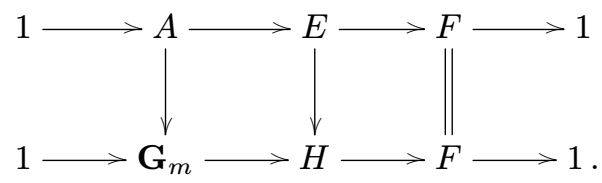

Comme le groupe $F$ est de type multiplicatif (il est fini commutatif et $k$ est de caractéristique 0), la flèche $H \rightarrow F$ du diagramme se factorise par une flèche $H^{\text {mult }} \rightarrow F$.

D'après [21] (preuve de la proposition 6.1), il existe $a \in H^{1}(k, \widehat{F})$ dont, pour une infinité de $v$, la localisation $a_{v} \in H^{1}\left(k_{v}, \widehat{F}\right)$ est non nulle, mais tel que pour presque toute place $v$ on a $a_{v}$ orthogonale à $I_{v}:=\operatorname{Im}\left[H^{1}\left(k_{v}, E\right) \rightarrow H^{1}\left(k_{v}, F\right)\right]$, laquelle contient $\operatorname{Im}\left[H^{1}\left(k_{v}, H\right) \rightarrow H^{1}\left(k_{v}, F\right)\right]$ car la flèche $H^{2}\left(k_{v}, A\right)=\left(\operatorname{Br} k_{v}\right)[p] \rightarrow H^{2}\left(k_{v}, \mathbf{G}_{m}\right)=$ $\operatorname{Br} k_{v}$ est injective. Soit alors $b$ l'image de $a$ dans $H^{1}\left(k, \widehat{H}^{\text {mult }}\right)$; soit $b_{v} \in H^{1}\left(k_{v}, \widehat{H}^{\text {mult }}\right)$ la localisation de $b$. Soit $c_{v}$ un élément de $\operatorname{Im}\left[H^{1}\left(k_{v}, H\right) \rightarrow H^{1}\left(k_{v}, H^{\text {mult }}\right)\right]$, notons $d_{v}$ l'image de $c_{v}$ dans $H^{1}\left(k_{v}, F\right)$. Comme $d_{v}$ est dans l'image de $H^{1}\left(k_{v}, H\right)$, on obtient d'après ce qu'on a vu plus haut que $d_{v}$ est aussi dans $I_{v}$. Comme on a, par fonctorialité, égalité des accouplements locaux $\left(b_{v}, c_{v}\right)$ et $\left(a_{v}, d_{v}\right)$, on obtient finalement pour presque toute place $v$ que $\left(b_{v}, c_{v}\right)=0$, et donc que $b_{v}$ est orthogonal à $\operatorname{Im}\left[H^{1}\left(k_{v}, H\right) \rightarrow H^{1}\left(k_{v}, H^{\text {mult }}\right)\right]$.

Par ailleurs, comme $F$ est l'abélianisé de $E$, la flèche associée $\widehat{F} \rightarrow \widehat{E}$ entre leurs groupes de caractères est un isomorphisme; comme elle se factorise par la flèche $\widehat{F} \rightarrow \widehat{H}^{\text {mult }}$ (via le diagramme commutatif ci-dessus), cette dernière admet une rétraction. En particulier la flèche induite $H^{1}\left(k_{v}, \widehat{F}\right) \rightarrow H^{1}\left(k_{v}, \widehat{H}^{\text {mult }}\right)$ est injective, ce qui implique que $b$ n'est pas dans $\amalg_{\omega}^{1}\left(\widehat{H}^{\text {mult }}\right)$ vu l'hypothèse sur $a$.

D'après la formule de compatibilité (voir théorème 6.2(b)) et le fait que la flèche d'évaluation $X\left(k_{v}\right) \rightarrow H^{1}\left(k_{v}, H\right)$ (associée au $H$-torseur $\mathrm{SL}_{n} \rightarrow X$ ) soit surjective (ceci résulte de ce que $H^{1}\left(k_{v}, \mathrm{SL}_{n}\right)=0$ ), on obtient alors que pour presque toute place $v$, on a $b\left(P_{v}\right)=0$ pour tout $k_{v}$-point $P_{v}$ de $X$, ce qui montre que $b \in \mathrm{Br}_{\mathrm{nr}} X$ via le théorème 2.2.1 de [31]. 
On peut également modifier légèrement la construction précédente pour vérifier que dans le cas d'un corps fini, l'hypothèse Pic $\bar{G}=0$ dans le théorème 7.5 (ii) est nécessaire (voir la remarque qui suit le théorème 7.5 ).

Proposition 8.5. - Il existe un corps fini $\mathbf{F}$ de caractéristique $p \geq 3$, un groupe semisimple connexe $G$ (de groupe fondamental $\mu_{2}$ ) sur $\mathbf{F}$ et un $\mathbf{F}$-sous-groupe $H$ de $G$, tel que $H$ soit un groupe fini constant commutatif d'ordre 16 , isomorphe à $(\mathbf{Z} / 2 \mathbf{Z})^{2} \times \mathbf{Z} / 4 \mathbf{Z}$, de sorte que si $X=G / H$, on a

$$
\left(\mathrm{Br}_{\mathrm{nr}}{ }_{1} X\right)\{2\} \neq 0 \text {. }
$$

Démonstration. - On remarque d'abord que dans la proposition 6.1 de [21], dans le cas d'un 2-groupe, on peut remplacer le groupe $E$ d'ordre 64 par le groupe fini $H_{0}$ d'ordre 32 dont une présentation est

$$
H_{0}=\left\langle x, y, z: x^{2}=y^{4}=z^{4}=1 ;[x, y]=z^{2}\right\rangle .
$$

Il s'agit par exemple du groupe d'ordre 32 de numéro 24 dans la classification de GAP.

La preuve de la proposition 6.1 dans [21] assure que pour toute $\mathbf{Q}$-représentation fidèle $\rho: H_{0} \rightarrow \mathrm{SL}_{n, \mathbf{Q}}$, le groupe $\left(\mathrm{Br}_{\mathrm{nr} 1} X_{\rho}\right)\{2\}$ est strictement plus gros que le sous-groupe de $\left(\operatorname{Br}_{1} X_{\rho}\right)\{2\}$ formé des éléments localement constants en presque toute place, avec $X_{\rho}:=\mathrm{SL}_{n, \mathbf{Q}} / \rho\left(H_{0}\right)$.

On considère la Q-représentation $\rho_{0}$ suivante : on définit $\rho_{0}: H_{0} \rightarrow \mathrm{SL}_{8, \mathbf{Q}}$ par les formules

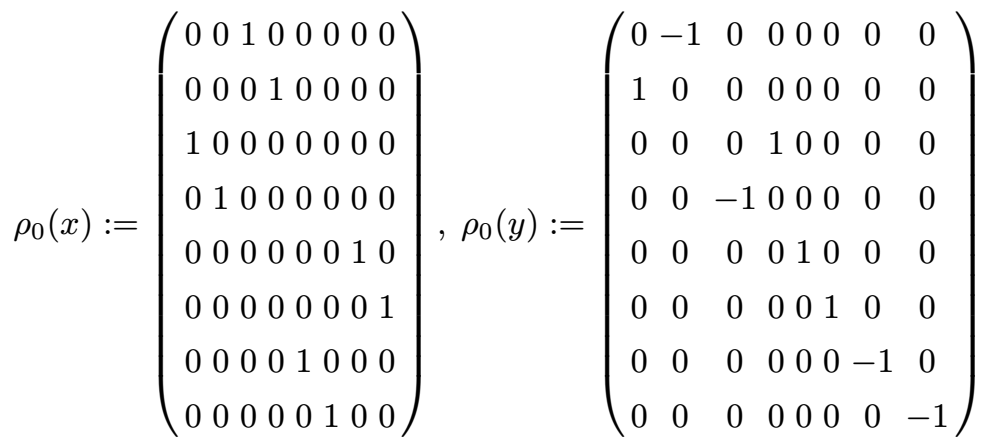

$$
\begin{aligned}
& \text { et } \rho_{0}(z):=\left(\begin{array}{cccccccc}
0 & -1 & 0 & 0 & 0 & 0 & 0 & 0 \\
1 & 0 & 0 & 0 & 0 & 0 & 0 & 0 \\
0 & 0 & 0 & -1 & 0 & 0 & 0 & 0 \\
0 & 0 & 1 & 0 & 0 & 0 & 0 & 0 \\
0 & 0 & 0 & 0 & 0 & -1 & 0 & 0 \\
0 & 0 & 0 & 0 & 1 & 0 & 0 & 0 \\
0 & 0 & 0 & 0 & 0 & 0 & 0 & -1 \\
0 & 0 & 0 & 0 & 0 & 0 & 1 & 0
\end{array}\right)
\end{aligned}
$$

On trouvera dans [12] une représentation complexe de $H_{0}$, très similaire à la représentation $\rho_{0}$ (dans [12], le groupe $H_{0}$ s'appelle $(16 ; 24)$ ).

On vérifie facilement que $\rho_{0}$ définit une représentation fidèle de $H_{0}$ dans $\mathrm{SL}_{8, \mathbf{Q}}$. 
Or $\rho_{0}\left(z^{2}\right)=-I_{8}$ est central dans $\mathrm{SL}_{8, \mathbf{Q}}$, donc on dispose du diagramme commutatif suivant, où $G:=\mathrm{SL}_{8, \mathbf{Q}} /\left\{ \pm I_{8}\right\}$ et $H:=H_{0}^{\text {ab }}=H /\left\langle z^{2}\right\rangle$ :

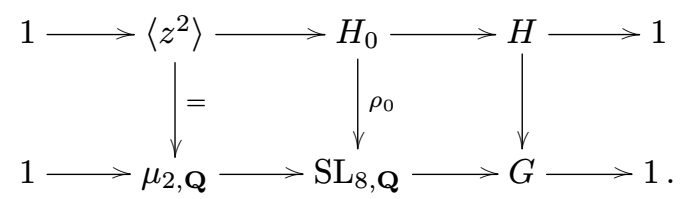

Ce diagramme permet d'identifier $X_{\rho_{0}}=\mathrm{SL}_{8, \mathbf{Q}} / H_{0}$ avec le quotient $G / H$ (comme $\mathbf{Q}$-variétés).

Enfin, on a bien $H \cong(\mathbf{Z} / 2 \mathbf{Z})^{2} \times \mathbf{Z} / 4 \mathbf{Z}$, et $G$ est linéaire connexe semi-simple, de groupe fondamental $\mu_{2}$.

On étend ensuite $H, G, \rho_{0}$ et $X_{\rho_{0}}$ sur un ouvert de $\operatorname{Spec} \mathbf{Z}$, de bonne réduction. Supposons que pour tout point fermé $s$ de cet ouvert, le groupe de Brauer non ramifié de la fibre de $X_{\rho_{0}}$ au-dessus de $s$ (qui est un espace homogène d'un groupe semi-simple connexe à stabilisateur $H$ sur le corps fini $k(s)$ ) soit trivial. Alors la preuve de la proposition 2 de [15] assure que $\mathrm{Br}_{\mathrm{nr} 1} X_{\rho_{0}}$ coïncide avec le sous-ensemble de $\operatorname{Br}_{1} X_{\rho_{0}}$ formés des éléments localement constants en presque toute place. Cela contredit la proposition $6.1 \mathrm{de}$ [21].

Donc il existe bien un corps fini $\mathbf{F}$ et un espace homogène sur $\mathbf{F}$ de la forme $X=G / H$, avec $G$ semi-simple (connexe) et $H$ abélien fini constant d'ordre 16 tels que $\left(\mathrm{Br}_{\mathrm{nr} 1} X\right)\{2\} \neq 0$.

Remarque. - La preuve assure que l'on peut aussi écrire $X$ sous la forme $X=\mathrm{SL}_{8, \mathbf{F}} / H_{0}$, où $H_{0}$ est un groupe fini constant d'ordre 32 . Cela fournit en particulier un exemple de groupe fini $H_{0}$ d'ordre 32 tel que le corps des fonctions de $X$ ne soit pas une extension transcendante pure du corps fini $\mathbf{F}$, et donne ainsi un contre-exemple au problème de Noether sur un corps fini. On notera par contre qu'on ne peut trouver un tel contre-exemple avec un 2-groupe $H_{0}$ sur un corps $\mathbf{F}$ de caractéristique 2 (d'où le fait qu'on ait pris $p \geq 3$ dans la proposition 8.5), car d'après un théorème de Kuniyoshi ([38]), la variété $X$ serait alors $\mathbf{F}$-rationnelle.

\section{Appendice A}

\section{Compatibilité pour un tore}

Dans cet appendice nous prouvons la proposition A.1 que nous utilisons dans la preuve du lemme 6.4.

Soit $T$ un tore sur un corps $k$. On pose $G=T, H=1, X=T$. Alors [10, thm. 7.2] donne un isomorphisme

$$
\mu_{\mathrm{BvH}}: \mathrm{Br}_{\mathrm{a}} T \stackrel{\sim}{\rightarrow} H^{1}(k,[\widehat{G} \rightarrow \widehat{H}])=H^{1}\left(k,[\widehat{T} \rightarrow 0]=H^{2}(k, \widehat{T}),\right.
$$

où $\mathrm{Br}_{\mathrm{a}} T=\mathrm{Br}_{1} T / \mathrm{Br} k$. 
Proposition A.1. - Le diagramme suivant est anti-commutatif :

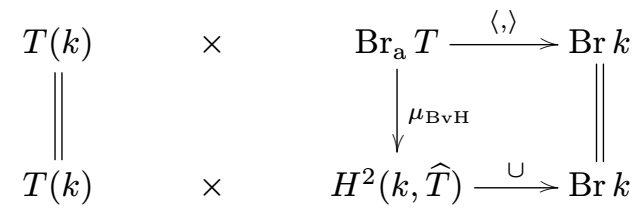

où l'accouplement supérieur est défini en utilisant les homomorphismes

$$
\mathrm{Br}_{\mathrm{a}} T \stackrel{\sim}{\rightarrow} \mathrm{Br}_{1, e} T \hookrightarrow \mathrm{Br}_{1} T .
$$

Pour prouver la proposition nous avons besoin d'une série de lemmes.

Lemme A.2. - Soit $X$ une $k$-variété lisse et géométriquement intègre munie d'un k-point m. On note $\bar{k}[X]^{*}:=H^{0}\left(\bar{X}, \mathbf{G}_{m}\right)$ le groupe multiplicatif des fonctions inversibles sur $X$ et $U(\bar{X}):=\bar{k}[X]^{*} / \bar{k}^{*}$. Alors le diagramme suivant est commutatif :

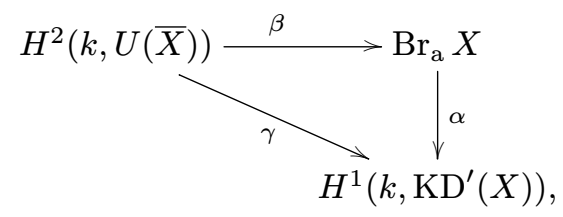

où $\beta$ est l'homomorphisme canonique de Sansuc [44, Lemme 6.3(ii)], $\alpha$ est l'isomorphisme canonique de [9, Cor. 2.20(i)], et $\gamma$ est l'homomorphisme canonique induit par le morphisme de complexes

$$
\delta_{\text {nat }}: U(\bar{X})=\mathscr{H}^{0}\left(\mathrm{KD}^{\prime}(X)[-1]\right) \hookrightarrow \mathrm{KD}^{\prime}(X)[-1],
$$

où $\mathcal{H}^{0}$ désigne la cohomologie du complexe en degré 0 .

Démonstration. - (1) Rappelons que $\mathbf{R} p_{*} \mathbf{G}_{m, X}$ est un certain complexe $\left[C^{0} \rightarrow C^{1}\right][-1]$ de $\operatorname{Gal}(\bar{k} / k)$-modules en degrés 0 et 1 (défini à un isomorphisme canonique près dans la catégorie dérivée) avec 0-cohomologie $\mathcal{H}^{0}\left(\mathbf{R} p_{*} \mathbf{G}_{m, X}\right)=\bar{k}[X]^{*}$. Le groupe $\bar{k}^{*}$ s'injecte canoniquement dans $\bar{k}[X]^{*}$, et on a

$$
\mathrm{KD}^{\prime}(X):=\left[C^{0} / \bar{k}^{*} \rightarrow C^{1}\right]
$$

La cohomologie en degré -1 du complexe $\mathrm{KD}^{\prime}(X)$ est donnée par

$$
\mathcal{H}^{-1}\left(\mathrm{KD}^{\prime}(X)\right)=U(\bar{X}):=\bar{k}[X]^{*} / \bar{k}^{*} .
$$

On note qu'il y a un isomorphisme canonique

$$
\alpha^{\prime}: \operatorname{Br}_{1} X \stackrel{\sim}{\rightarrow} H^{2}\left(k, \mathbf{R} p_{*} \mathbf{G}_{m, X}\right),
$$

voir [9, 2.18].

(2) On considère l'homomorphisme

$$
H^{2}\left(k, \bar{k}[X]^{*}\right) \rightarrow H^{2}\left(k, \mathbf{R} p_{*} \mathbf{G}_{m, X} \stackrel{\left(\alpha^{\prime}\right)^{-1}}{\longrightarrow} \mathrm{Br}_{1} X\right.
$$

où le premier homomorphisme est induit par le morphisme de complexes

$$
\bar{k}[X]^{*} \hookrightarrow \mathbf{R} p_{*} \mathbf{G}_{m, X},
$$

alors il résulte des définitions que la composition coïncide avec l'homomorphisme de Sansuc [44, lemme 6.3(i)].

$4^{\text {e }}$ SÉRIE - TOME $46-2013$ - No 4 
(3) On considère l'homomorphisme

$$
\beta: H^{2}(k, U(\bar{X})) \rightarrow \mathrm{Br}_{\mathrm{a}} X
$$

de Sansuc [44, lemme 6.3(ii)]. Cet homomorphisme est défini comme suit : le $k$-point $m \in X(k)$ définit une section homomorphique $\operatorname{Gal}(\bar{k} / k)$-équivariante $\sigma_{m}: U(\bar{X}) \rightarrow \bar{k}[X]^{*}$ de l'épimorphisme canonique $\bar{k}[X]^{*} \rightarrow U(\bar{X})$ donnée par

$$
\sigma_{m}([f])(x)=f(x) / f(m)
$$

pour la classe $[f]$ d'une fonction $f \in \bar{k}[X]^{*}$. Alors $\beta$ est la composition

$$
H^{2}(k, U(\bar{X})) \stackrel{\left(\sigma_{m}\right)_{*}}{\longrightarrow} H^{2}(k, \bar{k}[X]) \rightarrow H^{2}\left(k, \mathbf{R} p_{*} \mathbf{G}_{m, X}\right) \stackrel{\left(\alpha^{\prime}\right)^{-1}}{\longrightarrow} \operatorname{Br}_{1} X \rightarrow \mathrm{Br}_{\mathrm{a}} X .
$$

(4) On a un diagramme commutatif

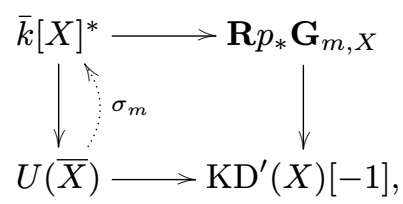

qui induit un diagramme commutatif d'hypercohomologie

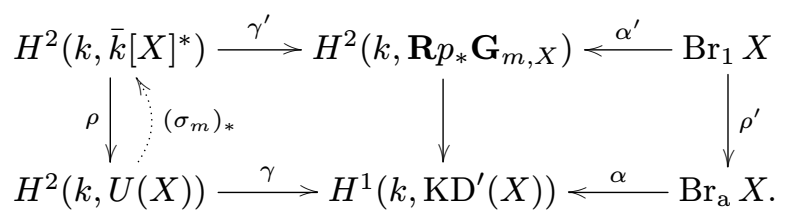

Comme $\beta=\rho^{\prime} \circ\left(\alpha^{\prime}\right)^{-1} \circ \gamma^{\prime} \circ\left(\sigma_{m}\right)_{*}$, on en conclut que $\gamma=\alpha \circ \beta$.

Lemme A.3. - Soient T, $G, H$ et $X$ comme au début de l'appendice; on désigne par

$$
\delta_{\mathrm{BvH}}: \widehat{T} \rightarrow\left[\bar{k}(\bar{X})^{*} / \bar{k}^{*} \rightarrow \operatorname{Div} \bar{X}\right][-1]
$$

l'isomorphisme dans la catégorie dérivée de [10, Thm. 5.8]. Alors $\delta_{\mathrm{BvH}}$ est l'opposé du quasiisomorphisme naturel

$$
\delta_{\text {nat }}: \widehat{T} \rightarrow\left[\bar{k}(\bar{X})^{*} / \bar{k}^{*} \rightarrow \operatorname{Div} \bar{X}\right][-1]
$$

induit par l'injection naturelle $\widehat{T}=U(\bar{X}) \hookrightarrow \bar{k}(\bar{X})^{*} / \bar{k}^{*}$, à savoir $\delta_{\mathrm{BvH}}=-\delta_{\text {nat }}$.

Démonstration. - On commence par considérer [10, diagramme (13) après le Thm. 4.10] :

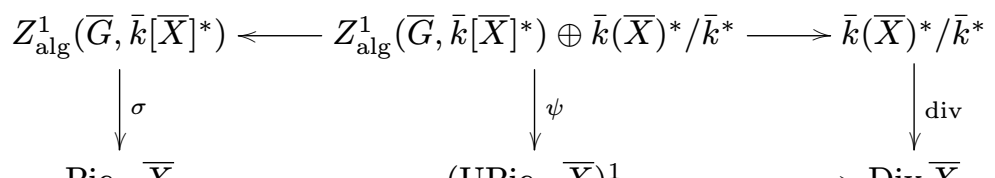

$\operatorname{Pic}_{G} \bar{X} \longleftrightarrow\left(\operatorname{UPic}_{G} \bar{X}\right)^{1} \longrightarrow \operatorname{Div} \bar{X}$

(voir [10] pour les notations), où la flèche $\psi$ est donnée par

$$
\psi(z,[f])=\left(z \cdot d^{0}(f), \operatorname{div}(f)\right) \in\left(\operatorname{UPic}_{G} \bar{X}\right)^{1} \subset Z_{\text {alg }}^{1}\left(\bar{G}, \bar{k}(\bar{X})^{*}\right) \oplus \operatorname{Div} \bar{X},
$$

et toutes les flèches non étiquetées sont les flèches évidentes.

On a $Z_{\text {alg }}^{1}\left(\bar{G}, \bar{k}[\bar{X}]^{*}\right)=\widehat{T}, \operatorname{Pic}_{G} \bar{X}=\widehat{H}=0$. On pose

$$
a=\mathrm{id}: \widehat{T} \stackrel{\sim}{\rightarrow} Z_{\text {alg }}^{1}\left(\bar{G}, \bar{k}[\bar{X}]^{*}\right) .
$$


Clairement, $a$ induit un morphisme de complexes

$$
a^{\prime}: \widehat{T} \rightarrow\left[Z_{\text {alg }}^{1}\left(\bar{G}, \bar{k}[\bar{X}]^{*}\right) \rightarrow \operatorname{Pic}_{G} \bar{X}\right][-1] .
$$

On considère l'injection canonique

$$
c: \widehat{T}=U(\bar{X}) \hookrightarrow \bar{k}(\bar{X})^{*} / \bar{k}^{*} .
$$

Clairement, $c$ induit un morphisme de complexes

$$
c^{\prime}=\delta_{\text {nat }}: \widehat{T} \rightarrow\left[\bar{k}(\bar{X})^{*} / \bar{k}^{*} \rightarrow \operatorname{Div} \bar{X}\right][-1] .
$$

On définit

$$
b: \widehat{T} \rightarrow Z_{\text {alg }}^{1}\left(\bar{G}, \bar{k}[\bar{X}]^{*}\right) \oplus \bar{k}(\bar{X})^{*} / \bar{k}^{*}
$$

$\operatorname{par} b(\chi)=(a(\chi),-c(\chi))$ pour $\chi \in \widehat{T}$. Alors il résulte de la formule pour $\psi$ que $\psi \circ \beta=0$ (on utilise la formule pour $b$ avec le signe opposé à $c(\chi)$ !), donc $b$ induit un morphisme de complexes

$$
b^{\prime}: \widehat{T} \rightarrow\left[Z_{\text {alg }}^{1}\left(\bar{G}, \bar{k}[\bar{X}]^{*}\right) \oplus \bar{k}(\bar{X})^{*} / \bar{k}^{*} \rightarrow\left(\operatorname{UPic}_{G} \bar{X}\right)^{1}\right][-1] .
$$

Clairement, on a un diagramme commutatif

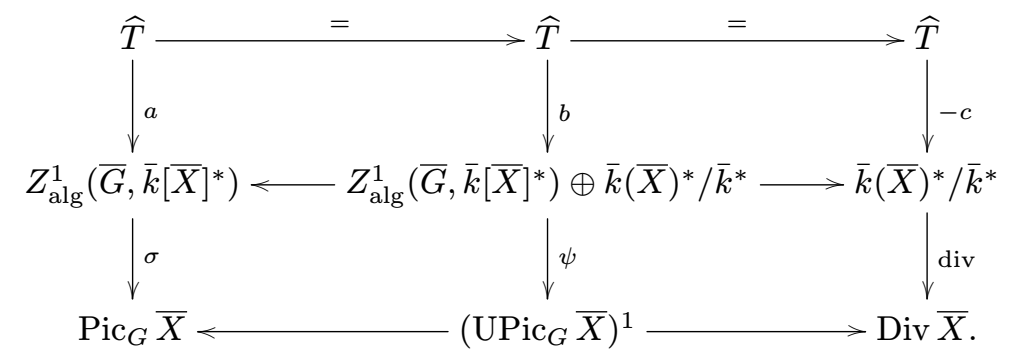

La partie inférieure du diagramme induit des isomorphismes (dans la catégorie dérivée) :

$$
\left[Z_{\mathrm{alg}}^{1}\left(\bar{G}, \bar{k}[\bar{X}]^{*}\right) \rightarrow \operatorname{Pic}_{G} \bar{X}\right] \simeq\left[Z_{\mathrm{alg}}^{1}\left(\bar{G}, \bar{k}[\bar{X}]^{*}\right) \oplus \bar{k}(\bar{X})^{*} / \bar{k}^{*} \rightarrow\left(\operatorname{UPic}_{G} \bar{X}\right)^{1}\right]
$$

et

$$
\left[Z_{\text {alg }}^{1}\left(\bar{G}, \bar{k}[\bar{X}]^{*}\right) \oplus \bar{k}(\bar{X})^{*} / \bar{k}^{*} \rightarrow\left(\operatorname{UPic}_{G} \bar{X}\right)^{1}\right] \simeq\left[\bar{k}(X)^{*} / \bar{k}^{*} \rightarrow \operatorname{Div} \bar{X}\right] .
$$

Or l'isomorphisme (dans la catégorie dérivée) $\delta_{\mathrm{BvH}}$ est la composition de $a^{\prime}$ et du composé des deux isomorphismes ci-dessus. On déduit du diagramme que $\delta_{\mathrm{BvH}}=-c^{\prime}$. Comme $\delta_{\text {nat }}=c^{\prime}$, on conclut que $\delta_{\mathrm{BvH}}=-\delta_{\text {nat }}$.

Lemme A.4. - On désigne par $\lambda_{\mathrm{S}}: H^{2}(k, \widehat{T}) \stackrel{\sim}{\rightarrow} \operatorname{Br}_{\mathrm{a}} T$ l'isomorphisme canonique de Sansuc [44, lemme 6.9]. Alors $\mu_{\mathrm{BvH}} \circ \lambda_{\mathrm{S}}=-\mathrm{id}: H^{2}(k, \widehat{T}) \rightarrow \mathrm{Br}_{\mathrm{a}} T \rightarrow H^{2}(k, \widehat{T})$.

Démonstration. - On a un diagramme commutatif

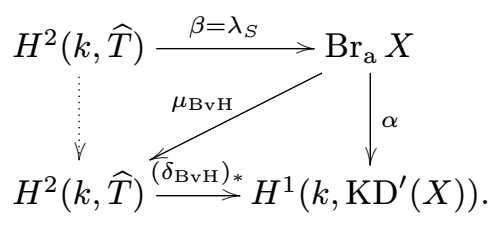

$4^{\mathrm{e}}$ SÉRIE - TOME $46-2013$ - No 4 
Par définition $\lambda_{S}=\beta, \mu_{\mathrm{BvH}}=\left(\delta_{\mathrm{BvH}}\right)_{*}^{-1} \circ \alpha$. D'après le lemme A.3, on a $\delta_{\mathrm{BvH}}=-\delta_{\text {nat. }}$. Par définition $\gamma=\left(\delta_{\text {nat }}\right)_{*}$, voir le lemme A.2. Donc

$$
\mu_{\mathrm{BvH}}=\left(-\delta_{\text {nat }}\right)_{*}^{-1} \circ \alpha=-\left(\delta_{\text {nat }}\right)_{*}^{-1} \circ \alpha=-\gamma^{-1} \circ \alpha .
$$

Ainsi

$$
\mu_{\mathrm{BvH}} \circ \lambda_{\mathrm{S}}=-\gamma^{-1} \circ \alpha \circ \beta=-\mathrm{id},
$$

parce que, d'après le lemme A.2, on a $\gamma=\alpha \circ \beta$.

Preuve de la proposition A.1. - On considère le diagramme

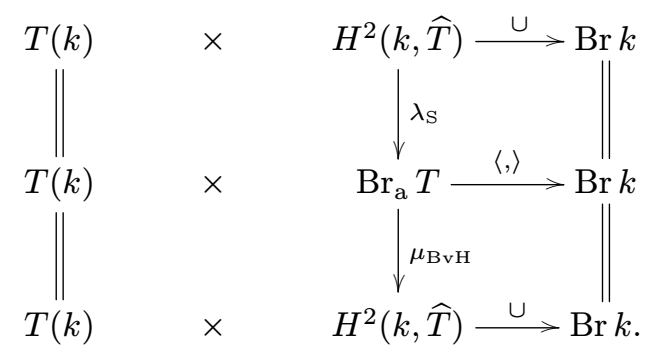

Le rectangle supérieur de ce diagramme est commutatif, grâce à [44, diagramme (8.11.2)]. D'autre part, si on supprime la ligne médiane de ce diagramme, alors le diagramme qui en résulte sera anti-commutatif, parce que d'après le lemme A.4 $\mu_{\mathrm{BvH}} \circ \lambda_{\mathrm{S}}=-\mathrm{id}$. On conclut que le rectangle inférieur du diagramme est anti-commutatif.

\section{Appendice B}

\section{Une autre preuve du théorème 6.2}

Dans cet appendice, nous donnons une preuve de l'expression explicite de $K D^{\prime}(X)$ et de la formule de compatibilité (théorème 6.2) indépendante de [10], sous l'hypothèse que le stabilisateur $H$ est de type (ssumult). Au passage, on établit quelques résultats généraux sur le complexe $K D^{\prime}$ associé à un torseur sous un groupe de type multiplicatif.

Dans toute la suite, $k$ est un corps et $p: X \rightarrow$ Spec $k$ désigne une variété lisse et géométriquement intègre sur $k$.

Lemme B.1. - Soit $[S \rightarrow T]$ un complexe de k-groupes de type multiplicatif. Soit $[\widehat{T} \rightarrow \widehat{S}]$ le complexe dual. Alors on a

$$
H_{\mathrm{fppf}}^{0}(X,[S \rightarrow T])=\operatorname{Hom}_{k}([\widehat{T} \rightarrow \widehat{S}], K D(X))
$$

où $\operatorname{Hom}_{k}(\ldots)$ désigne les homomorphismes dans la catégorie dérivée bornée $\mathscr{D}(k)$ des faisceaux étales sur $\operatorname{Spec} k$.

Pour simplifier les notations, on écrira souvent $H^{0}(X,[S \rightarrow T])$ pour $H^{0}\left(X,\left[p^{*} S \rightarrow p^{*} T\right]\right)$. 
Démonstration. - L'argument est le même que dans la proposition 1.1 de [32]. Pour tout complexe borné $C^{\bullet}$ (resp. $D^{\bullet}$ ) de faisceaux étales sur $\operatorname{Spec} k$ (resp. sur $X$ ), on a $\operatorname{Hom}_{k}\left(C^{\bullet}, p_{*} D^{\bullet}\right)=\operatorname{Hom}_{X}\left(p^{*} C^{\bullet}, D^{\bullet}\right)$. On en déduit que le foncteur $\mathbf{R H o m}_{X}\left(\left[p^{*} \widehat{T} \rightarrow p^{*} \widehat{S}\right],.\right)$ est le composé des foncteurs $\mathbf{R} p_{*}$ et $\mathbf{R H o m}{ }_{k}([\widehat{T} \rightarrow \widehat{S}])$, ce qui permet d'obtenir (pour tout $n \geq 0$ ) :

$$
\mathbf{R}^{n} \operatorname{Hom}_{k}\left([\widehat{T} \rightarrow \widehat{S}], \mathbf{R} p_{*} \mathbf{G}_{m, X}\right)=\mathbf{R}^{n} \operatorname{Hom}_{X}\left(\left[p^{*} \widehat{T} \rightarrow p^{*} \widehat{S}\right], \mathbf{G}_{m, X}\right) .
$$

Comme par définition $K D(X)=\tau_{\leq 1} \mathbf{R} p_{*} \mathbf{G}_{m, X}[1]$, le triangle exact

$$
\tau_{\leq 1} \mathbf{R} p_{*} \mathbf{G}_{m, X} \rightarrow \mathbf{R} p_{*} \mathbf{G}_{m, X} \rightarrow \tau_{\geq 2} \mathbf{R} p_{*} \mathbf{G}_{m, X} \rightarrow \tau_{\leq 1} \mathbf{R} p_{*} \mathbf{G}_{m, X}[1]
$$

donne d'autre part

$$
\operatorname{Hom}_{k}([\widehat{T} \rightarrow \widehat{S}], K D(X))=\mathbf{R}^{1} \operatorname{Hom}_{k}\left([\widehat{T} \rightarrow \widehat{S}], \mathbf{R} p_{*} \mathbf{G}_{m, X}\right)
$$

via le fait que $\tau_{\geq 2} \mathbf{R} p_{*} \mathbf{G}_{m, X}$ est acyclique en degré $<2$. Pour conclure il suffit alors de montrer la formule

$$
\mathbf{R}^{1} \operatorname{Hom}_{X}\left(\left[p^{*} \widehat{T} \rightarrow p^{*} \widehat{S}\right], \mathbf{G}_{m, X}\right)=H_{\mathrm{fppf}}^{0}(X,[S \rightarrow T])
$$

qu'on peut récrire

$$
H_{\mathrm{fppf}}^{0}(X,[S \rightarrow T])=\operatorname{Hom}_{X}\left(\left[p^{*} \widehat{T} \rightarrow p^{*} \widehat{S}\right], \mathbf{G}_{m, X}[1]\right)
$$

ce qui résulte du lemme 3.1.

Remarque. - Là encore, la même méthode montre que l'assertion reste vraie si on remplace $\operatorname{Hom}_{k}(\ldots)$ par $\operatorname{Hom}_{\mathscr{D}_{\mathrm{fppf}}(k)}(\ldots)$ dans le groupe de droite.

Proposition B.2. - Soit $[S \rightarrow T]$ un complexe de k-groupes de type multiplicatif. Soit $f: Y \rightarrow X$ un $S$-torseur. On suppose de plus que $Y$ est muni d'une trivialisation du torseur $Y \wedge^{S} T \rightarrow X$ obtenu par changement de groupe structural. Soit $[[Y]]$ la classe correspondante dans $H_{\mathrm{fppf}}^{0}(X,[S \rightarrow T])$ et $u \in \operatorname{Hom}_{k}([\widehat{T} \rightarrow \widehat{S}], K D(X))$ le morphisme associé comme dans le lemme B.1. On définit un morphisme $\mu:[\widehat{T} \rightarrow \widehat{S}] \rightarrow K D^{\prime}(X)$ dans $\mathscr{D}(k)$ en composant u avec le morphisme canonique $v: K D(X) \rightarrow K D^{\prime}(X)$. Alors on a, pour tout $a \in H^{1}(k,[\widehat{T} \rightarrow \widehat{S}])$, la formule

$$
r\left(p^{*} a \cup[[Y]]\right)=\mu_{*}(a) .
$$

Démonstration. - C'est tout à fait similaire à la preuve du théorème 1.4. de [32]. On a (via le lemme B.1 et sa preuve) un diagramme commutatif

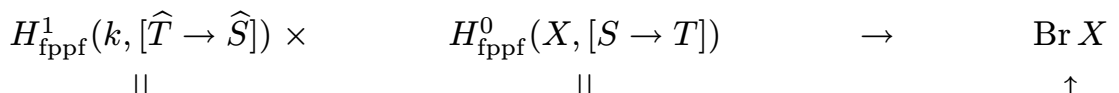

$$
\begin{aligned}
& H_{\text {fppf }}^{1}(k,[\widehat{T} \rightarrow \widehat{S}]) \times \operatorname{Hom}_{\mathscr{D}_{\mathrm{fppf}}(k)}\left([\widehat{T} \rightarrow \widehat{S}], \mathbf{R} p_{*} \mathbf{G}_{m, X}[1]\right) \rightarrow H_{\mathrm{fppf}}^{1}\left(k, \mathbf{R} p_{*} \mathbf{G}_{m, X}[1]\right) \\
& \begin{array}{ccc}
\| & \| & \uparrow \\
H^{1}(k,[\widehat{T} \rightarrow \widehat{S}]) \times \quad \operatorname{Hom}_{k}([\widehat{T} \rightarrow \widehat{S}], K D(X)) & \rightarrow & H^{1}(k, K D(X))
\end{array}
\end{aligned}
$$

où l'accouplement de la première ligne est donné par le cup-produit (cf. [40], Prop. V.1.20). On obtient donc, une fois qu'on a identifié $\mathrm{Br}_{1} X$ avec $H^{1}(k, K D(X))$ :

$$
p^{*} a \cup[[Y]]=u_{*}(a) .
$$

4 e SÉRIE - TOME $46-2013-$ No $^{\circ}$ 
Ainsi

$$
r\left(p^{*} a \cup[[Y]]\right)=r\left(u_{*}(a)\right)=v_{*}\left(u_{*}(a)\right)=\mu_{*}(a)
$$

par définition de $\mu$ et de $r$.

Rappelons la définition suivante ([32], section 2) :

Définition B.3. - Soit $V$ une $k$-variété (pas forcément intègre) munie d'une action $m: S \times V \rightarrow V$ d'un $k$-groupe de type multiplicatif $S$. On définit $\bar{k}[V]_{S}^{*}$ comme le sous-groupe de $\bar{k}[V]^{*}:=H^{0}\left(\bar{V}, \mathbf{G}_{m}\right)$ constitué des fonctions $f$ pour lesquelles il existe un caractère $\chi \in \widehat{S}$ vérifiant $m^{*} f=\chi \cdot f$ dans $H^{0}\left(\bar{S} \times \bar{V}, \mathbf{G}_{m}\right)$. De même si $\pi: Y \rightarrow X$ est un $S$-torseur, on définit le faisceau étale $\left(\pi_{*} \mathbf{G}_{m, Y}\right)_{S}$ comme le sous-faisceau de $\pi_{*} \mathbf{G}_{m, Y}$ tel que, pour tout morphisme étale $U \rightarrow X$, le groupe $\left(\pi_{*} \mathbf{G}_{m, Y}\right)_{S}(U)$ consiste en les $f: Y_{U} \rightarrow \mathbf{G}_{m, U}$ telles qu'il existe un caractère $\chi: S_{U} \rightarrow \mathbf{G}_{m, U}$ avec $m^{*} f=\chi \cdot f$, où $Y_{U}:=Y \times_{k} U$ et $S_{U}:=S \times_{k} U$.

Noter que si l'action du schéma en groupes $S$ sur $U(\bar{V})=\bar{k}[V]^{*} / \bar{k}^{*}$ est triviale (i.e. la flèche $m^{*}: H^{0}\left(\bar{V}, \mathbf{G}_{m}\right) / \bar{k}^{*} \rightarrow H^{0}\left(\bar{S} \times \bar{V}, \mathbf{G}_{m}\right) / \bar{k}^{*}$ induite par l'action $m: S \times V \rightarrow V$ coïncide avec la flèche induite par la projection $\bar{S} \times \bar{V} \rightarrow \bar{V}$ ), alors $\bar{k}[V]_{S}^{*}=\bar{k}[V]^{*}$. C'est en particulier le cas si $S$ est un tore et $V$ est géométriquement connexe ([32], remarque page 9).

Proposition B.4. - Soit $S$ un k-groupe de type multiplicatif lisse. Soit $\pi: Y \rightarrow X$ un $S$-torseur avec $Y$ géométriquement connexe. On suppose que l'action du schéma en groupes $S$ $\operatorname{sur} U(\bar{Y})=\bar{k}[Y]^{*} / \bar{k}^{*}$ est triviale.

Alors on a une suite exacte naturelle

$$
0 \rightarrow U(\bar{X}) \rightarrow U(\bar{Y}) \rightarrow \widehat{S} \rightarrow \operatorname{Pic} \bar{X} \rightarrow \operatorname{Pic} \bar{Y}
$$

où la flèche $\widehat{S} \rightarrow \operatorname{Pic} \bar{X}$ est le type du torseur $\pi$.

Démonstration. - On peut supposer $k=\bar{k}$. On note $T$ la composante connexe de l'identité dans $S$ et on pose $F:=S / T$ et $Z:=Y / T$. L'hypothèse que $S$ (et donc aussi $F$ ) est lisse implique alors que le $k$-groupe fini (et commutatif) $F$ est constant.

On a alors un diagramme commutatif de torseurs :

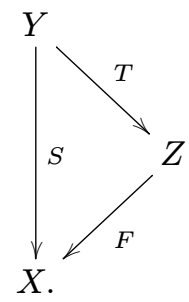


On en déduit le diagramme commutatif suivant :

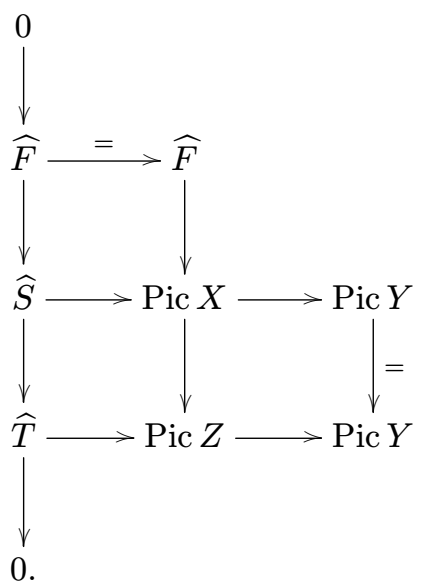

La première colonne est exacte, la ligne inférieure est exacte par [44], proposition 6.10. L'exactitude de la deuxième colonne est donnée par la suite exacte des termes de bas degré associée à la suite spectrale de Hochschild-Serre : $E_{2}^{p, q}:=H^{p}\left(F, H^{q}\left(Z, \mathbf{G}_{m}\right)\right) \Rightarrow$ $H^{p+q}\left(X, \mathbf{G}_{m}\right)$ du revêtement galoisien $Z \rightarrow X$. En effet $H^{0}\left(F, H^{1}\left(Z, \mathbf{G}_{m}\right)\right)$ est un sousgroupe de $\operatorname{Pic} Z$, et $H^{1}\left(F, H^{0}\left(Z, \mathbf{G}_{m}\right)\right)$ est un quotient de $H^{1}\left(F, k^{*}\right)=\widehat{F}$ via la suite exacte

$$
0 \rightarrow k^{*} \rightarrow H^{0}\left(Z, \mathbf{G}_{m}\right) \rightarrow U(Z) \rightarrow 0
$$

et l'hypothèse que le groupe fini $F$ agit trivialement sur $U(Y)$, donc aussi $\operatorname{sur} U(Z)$ (qui est un groupe abélien libre de type fini car $Z$ est lisse et connexe), ce qui implique $H^{1}(F, U(Z))=0$.

Une chasse au diagramme utilisant l'exactitude des deux premières colonnes et de la dernière ligne assure alors que la ligne centrale est exacte. Cela conclut la preuve, grâce à la proposition 2.5, (i) et (iii), de [32].

On rappelle le lemme suivant d'algèbre homologique (voir par exemple [23], lemme 2.3) :

Lemme B.5. - Soient $\mathscr{G}, \mathcal{B}$ deux catégories abéliennes et $F: \mathscr{G} \rightarrow \mathscr{B}$ un foncteur exact à gauche. On suppose que $\mathscr{G}$ a suffisamment d'injectifs. Soit $0 \rightarrow A \rightarrow B \rightarrow C \rightarrow 0$ une suite exacte d'objets de $\mathscr{G}$. Si le cobord $F(C) \rightarrow R^{1} F(A)$ est un épimorphisme, alors on a un isomorphisme canonique dans la catégorie dérivée :

$$
[F(B) \rightarrow F(C)] \rightarrow \tau_{\leq 1}(\mathbf{R} F)(A) .
$$

Démonstration. - C'est le lemme 2.3 de [23], dans le cas $n=1$.

Lemme B.6. - Soit $S$ un k-groupe de type multiplicatif lisse. Soit $\pi: Y \rightarrow X$ un $S$-torseur, avec $Y$ géométriquement connexe. On note $p: X \rightarrow$ Spec $k$ le morphisme structural et $q=(p \circ \pi): Y \rightarrow$ Spec $k$. On fait les deux hypothèses suivantes:

(a) L'action du schéma en groupes $S$ sur $U(\bar{Y})=\bar{k}[Y]^{*} / \bar{k}^{*}$ est triviale.

(b) On $a$ Pic $\bar{Y}=0$.

Alors on a un isomorphisme dans $\mathscr{D}(k)$ :

$$
\left[\bar{k}[Y]^{*} \rightarrow \widehat{S}\right] \rightarrow K D(X)
$$

$4^{\text {e }}$ SÉRIE - TOME $46-2013-$ No $^{\circ}$ 
où la flèche induite

$$
\widehat{S} \rightarrow K D(X)
$$

s'identifie à la classe $[Y] \in H_{\mathrm{fppf}}^{1}(X, S)=\operatorname{Hom}_{k}(\widehat{S}, K D(X))$ (au signe près).

Démonstration. - On a une suite exacte de faisceaux étales sur $X$ :

$$
0 \rightarrow \mathbf{G}_{m, X} \rightarrow\left(\pi_{*} \mathbf{G}_{m, Y}\right)_{S} \rightarrow p^{*} \widehat{S} \rightarrow 0
$$

(voir [32], proposition 2.5(i)). Notons que $p_{*} p^{*} \widehat{S}=\widehat{S}$ par connexité de $\bar{X}$. On applique le foncteur $p_{*}$ : la suite exacte de la proposition B.4 et l'hypothèse (b) assurent que l'on a une suite exacte de modules galoisiens

$$
0 \rightarrow p_{*} \mathbf{G}_{m, X} \rightarrow p_{*}\left(\pi_{*} \mathbf{G}_{m, Y}\right)_{S} \rightarrow \widehat{S} \rightarrow R^{1} p_{*} \mathbf{G}_{m, X} \rightarrow 0 .
$$

Alors le lemme B.5 assure que le morphisme naturel

$$
\left[p_{*}\left(\pi_{*} \mathbf{G}_{m, Y}\right)_{S} \rightarrow \widehat{S}\right] \rightarrow \tau_{\leq 1}\left(\mathbf{R} p_{*}\right) \mathbf{G}_{m, X}=K D(X)
$$

est un isomorphisme. Or l'hypothèse (a) assure que $p_{*}\left(\pi_{*} \mathbf{G}_{m, Y}\right)_{S}=\bar{k}[Y]^{*}$, d'où finalement un isomorphisme

$$
\left[\bar{k}[Y]^{*} \rightarrow \widehat{S}\right] \rightarrow K D(X) .
$$

Comme la classe $[Y] \in \operatorname{Ext}_{X}^{1}\left(\widehat{S}, \mathbf{G}_{m, X}\right)=\operatorname{Hom}_{X}\left(\widehat{S}, \mathbf{G}_{m, X}[1]\right)$ correspond au signe près ([32], Prop. 2.5(ii)) à l'extension donnée par (11), la flèche induite $\alpha: \widehat{S} \rightarrow K D(X)$ correspond bien à la classe $[Y]$ (cf. [32], appendice B).

Proposition B.7. - On garde les hypothèses et notations du lemme B.6. Soit T le k-tore dont le module des caractères est $\widehat{T}=\bar{k}[Y]^{*} / \bar{k}^{*}$ (qui est muni d'un morphisme canonique $\widehat{T} \rightarrow \widehat{S}$ par [32], Prop. 2.5).

(a) On a un isomorphisme dans $\mathscr{D}(k)$ :

$$
[\widehat{T} \rightarrow \widehat{S}] \rightarrow K D^{\prime}(X)
$$

(b) On suppose de plus que $Y$ possède un point rationnel $y \in Y(k)$. Alors y induit une trivialisation de $Y \wedge^{S} T$, telle que le morphisme $\mu$ associé à $Y$ comme dans la proposition B.2 soit un isomorphisme dans $\mathscr{D}(k)$.

Démonstration. - (a) L'assertion résulte du lemme B.6 : on a un isomorphisme $\mu_{0}:\left[\bar{k}[Y]^{*} \rightarrow \widehat{S}\right] \rightarrow K D(X)$ qui induit un isomorphisme $\bar{\mu}_{0}:[\widehat{T} \rightarrow \widehat{S}] \rightarrow K D^{\prime}(X)$.

(b) On dispose du diagramme commutatif (flèches rectilignes) suivant :

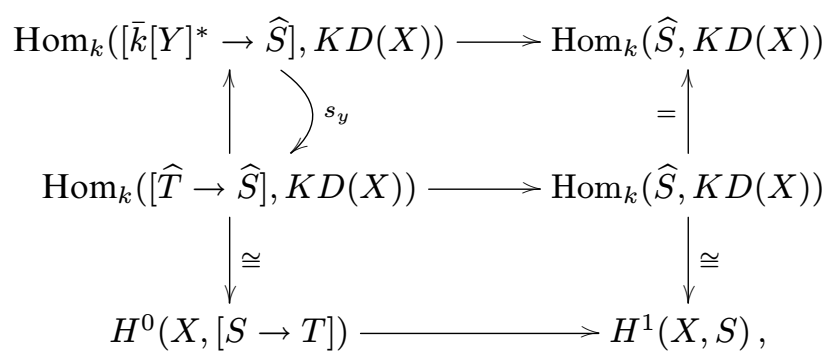

où $s_{y}$ est induite par la section de $\bar{k}[Y]^{*} \rightarrow \bar{k}[Y]^{*} / \bar{k}^{*}$ donnée par $y \in Y(k)$.

On note $\mu_{0, y}:=s_{y}\left(\mu_{0}\right):[\widehat{T} \rightarrow \widehat{S}] \rightarrow K D(X)$. 
Le diagramme précédent et la seconde partie du lemme B.6 assurent que l'image de $\mu_{0, y}$ dans $H^{0}(X,[S \rightarrow T])$ est la classe du torseur $Y \rightarrow X$ muni d'une trivialisation du torseur $Y \wedge^{S} T$, de sorte que le morphisme $[\widehat{T} \rightarrow \widehat{S}] \rightarrow K D^{\prime}(X)$ associé à cette trivialisation (comme à la proposition B.2) soit exactement $\bar{\mu}_{0}$. Cela assure le point (b) de la proposition.

Proposition B.8. - Soit G un k-groupe linéaire, réductif et connexe. Soit $H$ un k-sousgroupe de type (ssumult) de $G$, on pose $S=H^{\text {mult }}$ et $H^{\prime}=\operatorname{ker}\left[H \rightarrow H^{\text {mult }}\right]$. On suppose de plus que $\operatorname{Pic} \bar{G}=0$ et on pose $T=G^{\mathrm{tor}}$. On pose $X=G / H$ et on note $[[G]] \in H_{\mathrm{fppf}}^{0}(X,[H \rightarrow G])$ la classe induite par la trivialisation du X-torseur (sous $G$ ) $G \wedge^{H} G$ donnée par le neutre $e \in G(k)$. On note également

$$
\tau: X(k) \rightarrow H_{\mathrm{fppf}}^{0}(k,[H \rightarrow G]) \quad x \mapsto[[G]](x)
$$

la bijection donnée par l'évaluation.

Soit $Y:=G / H^{\prime}$, qu'on peut voir comme un $X$-torseur sous $S$ muni d'une trivialisation de $Y \wedge^{S} T$. Alors la flèche

$$
\mu:[\widehat{T} \rightarrow \widehat{S}] \rightarrow K D^{\prime}(X)
$$

associée (comme dans la proposition B.2) à Y est un isomorphisme dans $\mathscr{D}(k)$.

Démonstration. - On observe que comme

$$
\bar{k}[Y]^{*} / \bar{k}^{*}=\bar{k}[G]^{*} / \bar{k}^{*}=\widehat{G}=\widehat{T}
$$

par le lemme de Rosenlicht, l'action de $S$ sur $\bar{k}[Y]^{*} / \bar{k}^{*}$ est triviale. Comme $H$ est de type (ssumult), le groupe $H^{\prime}$ est connexe, lisse, et sans caractères; d'autre part $\bar{G}$ est une variété $\bar{k}$-rationnelle avec $\operatorname{Pic} \bar{G}=0$. Par [44], Prop. 6.10, on a alors $\operatorname{Pic} \bar{Y}=0$. Il suffit alors d'appliquer la proposition B.7(b) au $S$-torseur $Y \rightarrow X$.

Remarque. - Le même argument marche avec un $H$ un peu plus géneral (extension d'un groupe de type multiplicatif lisse par un groupe $H^{\prime}$ lisse, de composante neutre $H_{0}^{\prime}$ vérifiant : $H^{\prime} / H_{0}^{\prime}$ et $H_{0}^{\prime}$ sont sans caractères).

Theorème B.9. - On garde les hypothèses et notations de la proposition B.8. Soit

$$
\text { ab }: H_{\mathrm{fppf}}^{0}(k,[H \rightarrow G]) \rightarrow H_{\mathrm{fppf}}^{0}(k,[S \rightarrow T])
$$

l'application d'abélianisation et $\mathrm{ab}^{0}:=(\mathrm{ab} \circ \tau): X(k) \rightarrow H_{\mathrm{fppf}}^{0}(k,[S \rightarrow T])$.

Soit $\Phi_{X}: \operatorname{Br}_{1, e} X \rightarrow H^{1}(k,[\widehat{T} \rightarrow \widehat{S}])$ l'isomorphisme défini par $\Phi_{X}=\left(\mu_{*}\right)_{-1} \circ r$. Alors on a (au signe près) :

$$
\phi_{X}(\alpha) \cup \mathrm{ab}^{0}(x)=\alpha(x)
$$

pour tout $x \in X(k)$ et tout $\alpha \in \mathrm{Br}_{1, e} X$.

$4^{\mathrm{e}}$ SÉRIE - TOME $46-2013-\mathrm{N}^{\circ} 4$ 
Démonstration. - D’après la proposition B.2, on a

$$
\Phi_{X}\left(p^{*} a \cup[[Y]]\right)=\mu_{*}^{-1}\left(r\left(p^{*} a \cup[[Y]]\right)\right)=a
$$

pour tout $a \in H^{1}(k,[\widehat{T} \rightarrow \widehat{S}])$. Posons $a=\Phi_{X}(\alpha)$. Comme $\Phi_{X}$ est injective, on obtient

$$
p^{*}\left(\Phi_{X}(\alpha)\right) \cup[[Y]]=\alpha
$$

d'où en évaluant en $x$ et en remarquant que $[[Y]](x)=\mathrm{ab}(\tau(x))=\mathrm{ab}^{0}(x)$ :

$$
\Phi_{X}(\alpha) \cup \mathrm{ab}^{0}(x)=\alpha(x)
$$

qui est la formule voulue.

\section{Remerciements}

Les auteurs tiennent à remercier chaleureusement J.-L. Colliot-Thélène, C. D. GonzálezAvilés, B. Kahn et $\mathrm{O}$. Wittenberg pour d'intéressantes discussions pendant la préparation de cet article. Nous remercions également le rapporteur pour sa lecture attentive du manuscrit et ses judicieuses remarques.

\section{BIBLIOGRAPHIE}

[1] F. A. Bogomolov, Brauer groups of the fields of invariants of algebraic groups, Mat. Sb. 180 (1989), 279-293; traduction anglaise : Math. USSR Sb. 66 (1990), 285-299.

[2] A. Borel, Linear algebraic groups, 2e éd., Graduate Texts in Math. 126, Springer, 1991.

[3] M. V. Borovor, Abelianization of the second nonabelian Galois cohomology, Duke Math. J. 72 (1993), 217-239.

[4] M. V. Borovor, The Brauer-Manin obstructions for homogeneous spaces with connected or abelian stabilizer, J. reine angew. Math. 473 (1996), 181-194.

[5] M. V. Borovor, Abelian Galois cohomology of reductive groups, Mem. Amer. Math. Soc. 132 (1998).

[6] M. V. Borovoi, On the unramified Brauer group of a homogeneous space, prépublication arXiv:1206.1023.

[7] M. V. Borovoi, J.-L. Colliot-Thélène, A. Skorobogatov, The elementary obstruction and homogeneous spaces, Duke Math. J. 141 (2008), 321-364.

[8] M. V. Borovoi, C. Demarche, Manin obstruction to strong approximation for homogeneous spaces, Comment. Math. Helv. 88 (2013), 1-54.

[9] M. V. Borovoi, J. van Hamel, Extended Picard complexes and linear algebraic groups, J. reine angew. Math. 627 (2009), 53-82.

[10] M. V. Borovoi, J. van Hamel, Extended equivariant Picard complexes and homogeneous spaces, Transform. Groups 17 (2012), 51-86.

[11] F. Bruhat, J. Tits, Groupes algébriques sur un corps local. Chapitre III. Compléments et applications à la cohomologie galoisienne, J. Fac. Sci. Univ. Tokyo Sect. IA Math. 34 (1987), 671-698. 
[12] H. Chu, S.-J. Hu, M.-C. Kang, Y. G. Prokhorov, Noether's problem for groups of order 32, J. Algebra 320 (2008), 3022-3035.

[13] J.-L. Colliot-ThéLÈne, Birational invariants, purity and the Gersten conjecture, in $K$-theory and algebraic geometry : connections with quadratic forms and division algebras (Santa Barbara, CA, 1992), Proc. Sympos. Pure Math. 58, Amer. Math. Soc., 1995, 1-64.

[14] J.-L. COLliot-ThÉLÈne, Résolutions flasques des groupes linéaires connexes, J. reine angew. Math. 618 (2008), 77-133.

[15] J.-L. Colliot-Thélène, B. È. Kunyavskĭ̌, Groupe de Brauer non ramifié des espaces principaux homogènes de groupes linéaires, J. Ramanujan Math. Soc. 13 (1998), 37-49.

[16] J.-L. Colliot-Thélène, B. È. KunyavskiĬ, Groupe de Picard et groupe de Brauer des compactifications lisses d'espaces homogènes, J. Algebraic Geom. 15 (2006), 733-752.

[17] J.-L. Colliot-ThéLÈne, J.-J. SAnsuc, The rationality problem for fields of invariants under linear algebraic groups (with special regards to the Brauer group), in Algebraic groups and homogeneous spaces, Tata Inst. Fund. Res. Stud. Math., Tata Inst. Fund. Res., 2007, 113-186.

[18] J.-L. Colliot-Thélène, F. Xu, Brauer-Manin obstruction for integral points of homogeneous spaces and representation by integral quadratic forms, Compositio Math. 145 (2009), 309-363.

[19] B. Conrad, Deligne's notes on Nagata compactifications, J. Ramanujan Math. Soc. 22 (2007), 205-257.

[20] C. Demarche, Méthodes cohomologiques pour l'étude des points rationnels sur les espaces homogènes, thèse de doctorat, Université Paris-Sud, 2009.

[21] C. Demarche, Groupe de Brauer non ramifié d'espaces homogènes à stabilisateurs finis, Math. Ann. 346 (2010), 949-968.

[22] C. Demarche, Suites de Poitou-Tate pour les complexes de tores à deux termes, Int. Math. Res. Not. 2011 (2011), 135-174.

[23] C. Demarche, Une formule pour le groupe de Brauer algébrique d'un torseur, J. Algebra 347 (2011), 96-132.

[24] C. Demarche, Abélianisation des espaces homogènes et applications arithmétiques, in Torsors, étale homotopy and applications to rational points, LMS Lecture Notes $\mathbf{4 0 5}$, Cambridge Univ. Press, 2013.

[25] J.-C. DouaI, 2-cohomologie galoisienne des groupes semi-simples définis sur les corps locaux, C. R. Acad. Sci. Paris 280 (1975), 321-323.

[26] J.-C. DouaI, 2-cohomologie galoisienne des groupes semi-simples, thèse de doctorat, Université de Lille 1, 1976, Éditions universitaires européennes, Sarrebruck, 2010.

[27] J. Giraud, Cohomologie non abélienne, Grundl. math. Wissens. 179, Springer, 1971.

[28] C. D. González-Avilés, Quasi-abelian crossed modules and nonabelian cohomology, J. Algebra 369 (2012), 235-255.

[29] A. Grothendieck, Fondements de la géométrie algébrique. [Extraits du Séminaire Bourbaki, 1957-1962.], Secrétariat mathématique, 1962.

$4^{\mathrm{e}}$ SÉRIE - TOME $46-2013-\mathrm{N}^{\circ} 4$ 
[30] A. Grothendieck, Le groupe de Brauer, I, II, III, in Dix exposés sur la cohomologie des schémas, North-Holland \& Masson, 1968.

[31] D. Harari, Méthode des fibrations et obstruction de Manin, Duke Math. J. 75 (1994), 221-260.

[32] D. Harari, A. Skorobogatov, Descent theory for open varieties, in Torsors, étale homotopy and applications to rational points, LMS Lecture Notes 405, Cambridge Univ. Press, 2013.

[33] D. Harari, T. Szamuely, Arithmetic duality theorems for 1-motives, J. reine angew. Math. 578 (2005), 93-128.

[34] G. Harder, Halbeinfache Gruppenschemata über Dedekindringen, Invent. Math. 4 (1967), 165-191.

[35] R. Hartshorne, Algebraic geometry, Graduate Texts in Math. 52, Springer, 1977.

[36] L. Illusie, Y. Laszlo, F. Orgogozo, Travaux de Gabber sur l'uniformisation locale et la cohomologie étale des schémas quasi-excellents, Séminaire à l'École polytechnique 2006-2008, en préparation.

[37] M. Kneser, Galois-Kohomologie halbeinfacher algebraischer Gruppen über p-adischen Körpern. I, Math. Z. 88 (1965), 40-47; II, Math. Z. 89 (1965), 250-272.

[38] H. Kuniyoshi, On a problem of Chevalley, Nagoya Math. J. 8 (1955), 65-67.

[39] B. Margaux, Vanishing of Hochschild cohomology for affine group schemes and rigidity of homomorphisms between algebraic groups, Doc. Math. 14 (2009), 653672.

[40] J. S. Milne, Étale cohomology, Princeton Mathematical Series 33, Princeton Univ. Press, 1980.

[41] J. S. Milne, Arithmetic duality theorems, 2e éd., BookSurge, LLC, Charleston, SC, 2006.

[42] J. Oesterlé, Nombres de Tamagawa et groupes unipotents en caractéristique $p$, Invent. Math. 78 (1984), 13-88.

[43] M. Rosen, Number theory in function fields, Graduate Texts in Math. 210, Springer, 2002.

[44] J.-J. SAnsuc, Groupe de Brauer et arithmétique des groupes algébriques linéaires sur un corps de nombres, J. reine angew. Math. 327 (1981), 12-80.

[45] J-P. Serre, On the fundamental group of a unirational variety, J. London Math. Soc. 34 (1959), 481-484.

[46] J-P. Serre, Zeta and $L$ functions, in Arithmetical Algebraic Geometry (Proc. Conf. Purdue Univ., 1963), Harper \& Row, 1965, 82-92.

[47] J-P. Serre, Cohomologie galoisienne, 5e éd., Lecture Notes in Math. 5, Springer, 1994.

[48] A. Sкоrobogatov, Torsors and rational points, Cambridge Tracts in Mathematics 144, Cambridge Univ. Press, 2001.

[49] T. A. Springer, Nonabelian $H^{2}$ in Galois cohomology, in Algebraic Groups and Discontinuous Subgroups (Proc. Sympos. Pure Math., Boulder, Colo., 1965), Amer. Math. Soc., 1966, 164-182.

[50] C. A. Weibel, An introduction to homological algebra, Cambridge Studies in Advanced Math. 38, Cambridge Univ. Press, 1994. 
(Manuscrit reçu le 30 avril 2012; accepté, après révision, le 18 octobre 2012.)

Mikhail Borovor

Raymond and Beverly Sackler School of Math. Sciences
Tel Aviv University
69978 Tel Aviv, Israel
E-mail: borovoi@post.tau. ac.il
Cyril DemARCHE
Institut de Mathématiques de Jussieu
Université Pierre et Marie Curie
4 place Jussieu
75252 Paris Cedex 05, France
E-mail: demarche@math.jussieu.fr
David HARARI
Université Paris-Sud
Laboratoire de mathématiques
Bâtiment 425
F-91405 Orsay Cedex, France
E-mail: david.harari@math.u-psud.fr

